\title{
Theoretical study of constant current scanning tunneling spectroscopy in $\mathrm{Pb}$ overlayers
}

\author{
A. Zugarramurdi, ${ }^{1,2}$ N. Zabala, ${ }^{1,2,3}$ A. G. Borisov, ${ }^{4}$ and E. V. Chulkov ${ }^{2,3,5}$ \\ ${ }^{1}$ Elektrizitatea eta Elektronika Saila, Zientzia eta Teknologia Fakultatea, UPV/EHU, 644 P.K., ES-48080 Bilbao, Spain \\ ${ }^{2}$ Centro de Física de Materiales CFM - Materials Physics Center MPC, Centro Mixto CSIC-UPVIEHU, \\ ES-20018 San Sebastian/Donostia, Spain \\ ${ }^{3}$ Donostia International Physics Center (DIPC), P. de Manuel Lardizabal 4, ES-20018 San Sebastián/Donostia, Spain \\ ${ }^{4}$ Institut des Sciences Moléculaires d'Orsay, ISMO, UMR 8214 CNRS-Université Paris-Sud, Bât. 351, Université Paris-Sud, \\ FR-91405 Orsay CEDEX, France \\ ${ }^{5}$ Dpto. de Física de Materiales, Facultad de Ciencias Químicas, Universidad del País Vasco UPV/EHU, Apto. 1072, \\ ES-20080 San Sebastián/Donostia, Spain
}

(Received 17 June 2011; published 16 September 2011)

\begin{abstract}
We present a theoretical study of the constant current scanning tunneling spectroscopy of quantum well states localized in $\mathrm{Pb}(111)$ overlayers on $\mathrm{Cu}(111)$ surfaces. The distance-voltage characteristic of the tunneling junction is obtained with a mixed approach. The wave packet propagation technique is applied to describe electron tunneling from the tip into the sample, and the density functional calculations provide the necessary inputs for the one-dimensional model potential representation of the system. The excited-state population decay mechanisms via inelastic electron-electron and electron-phonon interactions are taken into account with a bias-dependent absorbing potential introduced in the metal. Our results are in good agreement with recent experimental studies [Phys. Rev. Lett. 102, 196102 (2009), Phys. Rev. B 81, 205438 (2010)] over the energy range where the free-electron description of the $\mathrm{Pb}$ overlayer used here applies. We find that at high bias the quantum well states experience a Stark energy shift and partially acquire a character of field emission resonances. The present model study also sheds light at the experimentally observed departure of the energies of the quantum well states from the particle-in-a-box prediction for the bias above $4 \mathrm{eV}$. The measured trend can be consistently explained as due to the departure of the realistic $\mathrm{Pb}$ band structure in the $\Gamma$-L direction from free-electron parabola when the electron momentum approaches the $\Gamma$ point.
\end{abstract}

DOI: 10.1103/PhysRevB.84.115422

PACS number(s): 73.63.Hs, 73.21.Fg, 79.70.+q, 68.37.Yz

\section{INTRODUCTION}

Electron confinement at metal surfaces is a fascinating research subject, as it addresses the fundamental issues developed in quantum mechanical textbooks and, at the same time, it holds a promise of engineering the surfaces with sought electronic and magnetic properties, which is of evident practical interest. ${ }^{1}$ Nowadays, structures allowing confinement in three dimensions (3D) (quantum corrals, ${ }^{2-4}$ adatom and vacancy islands, ${ }^{5-9}$ and self-organized molecular structures ${ }^{10}$ ) and two dimensions (2D) (atomic chains, ${ }^{1-13}$ and ad-molecule stripes ${ }^{14}$ ) have been designed and studied. Many of the above systems are accessible only owing to the scanning tunneling microscopy (STM). At variance, the quantum well states (QWSs) formed by one-dimensional (1D) electron confinement in thin metal overlayers can be studied with photoemission and inverse photoemission so that they are a subject of intense research activity already for decades. ${ }^{15-19}$

QWSs have been observed experimentally in a variety of layered metallic films on different substrates where potential barriers at the film/vacuum and film/substrate interfaces restrict the electron motion. The latter assumes that the reflectivity of the film/substrate interface is high. This is the case when, e.g., the substrate possesses a projected band gap in the direction perpendicular to the surface like the $\mathrm{L}$ gap for the (111) surfaces of noble metals. The bulk electronic structure of the film material evolves then into a set of subbands characterized by the quantized electron motion perpendicular to the film, and bulklike dispersion in the film plane. ${ }^{20-23}$
The QWSs can thus be readily understood in terms of the discrete level structure of a 1D potential well. In particular, the phase accumulation model ${ }^{24-26}$ appears extremely efficient in describing the energies of the QWSs at $\bar{\Gamma}$ point. ${ }^{15-17}$ When the one-electron tunneling into the substrate is possible along the direction perpendicular to the film, the QWSs turn into the quasistationary states or quantum well resonances (QWRs). ${ }^{27}$ The quasiclassical description in this case can be performed with a Fabry-Perot-type approach. ${ }^{15,28,29}$

Since the size $D$ of the confining box can only be changed by the discrete amount given by the interlayer distance $a$ in the metal film, the energies of the QWSs at $\bar{\Gamma}$ point show an oscillatory pattern with film thickness. ${ }^{15-17}$ This results in a quantum size effect, i.e., oscillations in the film-thickness dependence of the work function, ${ }^{30}$ chemical reactivity, ${ }^{31}$ electron-phonon coupling, ${ }^{32-34}$ surface energy, ${ }^{35,36}$ etc. Among different metals, $\mathrm{Pb}(111)$ overlayers (or extended nanoislands) grown on metallic and semiconductor substrates appear to be one of the most studied systems. Here, the Fermi wavelength in the $\langle 111\rangle$ direction is of the order of $4 a / 3\left(a=5.41 a_{0}\right)$. Then many of the overlayer properties show the quantum size effect with a two monolayer (ML) period..$^{30-32,35-37}$ Along with crystal and electronic structure studies, a number of recent experimental works focused on the characterization of the excited electron dynamics in $\mathrm{Pb}$ quantum wells. The timeresolved two-photon photoemission (TR-2PPE) technique has been used for supported $\mathrm{Pb}$ films ${ }^{23,38,39}$ as well as the scanningtunneling spectroscopy (STS) has been performed on large well characterized $\mathrm{Pb}$ adislands. ${ }^{22,40,41}$ 
From the theoretical side, only few works explicitly account for the substrate. ${ }^{35}$ The simpler and more often used strategy consists in discussing the overlayer properties based on the ab initio calculations performed for $\mathrm{Pb}$ slabs. ${ }^{34,42,43}$ A similar approach, avoiding complete ab initio account of the substrate has been adopted for the studies of excited electron dynamics in $\mathrm{Pb} / \mathrm{Si}(111) \mathrm{QWSs}$. The calculation of the electron-electron many-body decay rate has been restricted to bulk $\mathrm{Pb}, 39,41,44$ and the electron-phonon (e-ph) decay rate has been adopted from the studies of free-standing $\mathrm{Pb}$ slabs. ${ }^{41}$ In general, the inclusion of the substrate makes the ab initio computations extremely demanding. Thus it is desirable to explore the model approaches. With a price of loosing an exact atomistic description, these allow one to explicitly account for the overlayer-substrate system.

Thin $\mathrm{Pb}(111)$ overlayers grown on metallic surfaces, such as $\mathrm{Cu}(111)$ and $\operatorname{Ag}(111)$, are well-suited systems for their description by simple models. Indeed, close to the Fermi level the $\mathrm{Pb}$ band dispersion in the direction perpendicular to the overlayer surface has free-electron character. Moreover, the projected $\mathrm{L}$ gap of the $\mathrm{Cu}(111)$ and $\mathrm{Ag}(111)$ substrates is well described within the nearly-free-electron model ${ }^{45}$ (we show the relevant electronic structure in Fig. 1). The 1D pseudopotentials for the whole overlayer-substrate system have thus been set and used successfully to calculate structural and dynamic properties of QWSs in $\mathrm{Pb} / \mathrm{Cu}(111)$ at different coverages. A number of topics have been addressed, such as the electron confinement in the overlayers due to the projected band gap in $\Gamma$-L direction, the magic height distributions at different coverages, and the lifetimes of QWSs and quantum well resonances (QWRs). ${ }^{37,48-50}$

The present study extends our recent work $^{50}$ on the energies and lifetimes of the QWSs and QWRs in $\mathrm{Pb} / \mathrm{Cu}(111)$. We address theoretically recent STM and STS experiments on confined states in large $\mathrm{Pb}(111)$ islands deposited on $\mathrm{Cu}(111)$ and $\operatorname{Ag}(111)$ surfaces. ${ }^{21,22}$ These STS studies were performed at constant current regime, so that high-energy QWSs could be sampled as compared to previous works. ${ }^{51}$
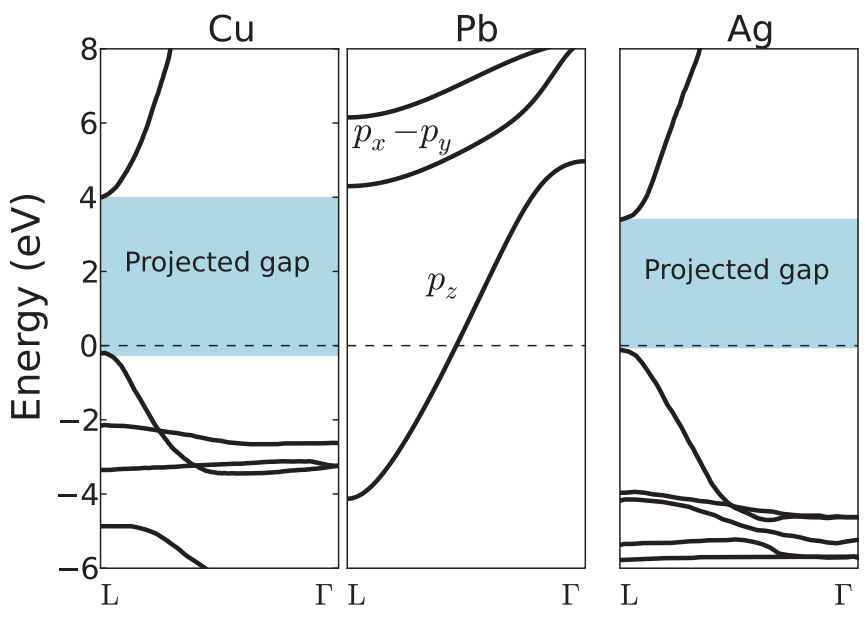

FIG. 1. (Color online) Electronic band structure of bulk $\mathrm{Cu},{ }^{47}$ $\mathrm{Pb},{ }^{41,44,46}$ and $\mathrm{Ag}^{47}$ along $\Gamma-\mathrm{L}$ direction. Energies are given with respect to the Fermi level. The shaded regions in $\mathrm{Cu}$ and $\mathrm{Ag}$ represent the projected band gap providing the confinement barrier in the substrate for the QWSs in Pb overlayers.
When the lateral extension of the $\mathrm{Pb}$ island is large enough, the quantization in the plane parallel to the surface resulting from the reflection by the island boundaries can be neglected because of the lifetime broadening. It is the quantization of the electron motion perpendicular to the surface that determines the discrete energy spectrum, so that the system is representative for the QWSs of the complete overlayer. The STM/STS studies have the advantage of dealing with wellcharacterized systems, where the information is not averaged over possible structural variations. This is why it has become a valuable complementary tool to photoemission and inverse photoemission techniques allowing to gain information on the band structure, ${ }^{52}$ quasiparticle lifetimes, ${ }^{18,53-55}$ phase-shifts for impurity or defect scattering, ${ }^{56}$ etc. On the other hand, and in particular for high bias, the electric field in the STM junction can a priori modify the energies of the electronic states at surfaces, so that the technique is not noninvasive. A classical example of this effect is the evolution of the image potential states (ISs) of the pristine metal surface ${ }^{24,57,58}$ into the field emission resonances (FERs) of the STM junction. ${ }^{59-66}$

The point raised above is one of the central issues of the present study: to what extent the QWSs are modified by the applied bias in the STM junction? Once this question is answered, we could address the role of the realistic $\mathrm{Pb}$ band structure in the experimentally observed trends. In this respect, present work deepens and extends our recent contribution on the subject. ${ }^{67}$ The flat-tip approximation is used here within the $1 \mathrm{D}$ model potential description of the STM junction (adlayer+substrate+tip) designed on the basis of the density-functional theory (DFT) calculations performed for $\mathrm{Pb} / \mathrm{Cu}(111)$. The constant current $d Z / d V$ spectra are calculated with the wave-packet-propagation (WPP) treatment of the electron transmission from the tip into the sample. We have found that for a broad range of overlayer thicknesses, the present model calculations reproduce the experimental data for the QWSs within the energy range where the free-electron description of $\mathrm{Pb}$ holds. Based on comparison between experimental data and theoretical calculations performed with and without biased STM tip, it is shown that at high bias, the energies of the QWSs are strongly affected by the tip-induced electric field. With the above findings, the measured departure of the QWSs energies from the particle-in-a-box prediction in the bias range of $4<V<4.5 \mathrm{eV}$ is explained as due to the realistic $\mathrm{Pb}$ band structure in $\Gamma$-L direction.

The paper is organized as follows. In Sec. II, we discuss the theoretical model used for the representation of the $\mathrm{Pb} / \mathrm{Cu}(111)$ + STM tip system and the wave-packet-propagation technique used for calculations of the $Z-V$ characteristics. In Sec. III, we present the results and their comparison with available experimental data. Finally, in Sec. IV, we give a summary of the work and conclusions. Atomic units are used throughout the paper unless otherwise stated.

\section{METHODS}

\section{A. Wave-packet-propagation (WPP) method}

The WPP is a one-electron time-domain approach based on the direct solution of the time-dependent Schrödinger equation. Nowadays, it has become a standard tool in quantum 
chemistry and physics allowing one to treat the response of a system to the time-dependent perturbation as well as to characterize its static properties such as energies and lifetimes of the electronic states and the scattering matrix. ${ }^{68}$ Here, we use the implementation of this method, as it has been developed by some of the coauthors to treat the dynamics of the excited states at surfaces. ${ }^{69}$

In brief, the dynamics of the "probe" electron in the system comprising a $\mathrm{Pb}$ layer adsorbed on the $\mathrm{Cu}(111)$ surface and the STM tip is studied. The system is described with a model $1 \mathrm{D}$ potential $\mathbb{U}(z)$, a function only of the $z$ coordinate perpendicular to the surface. It is derived from the density-functional theory calculations within the local-density approximation (LDA), as explained in the next section. The flat-tip approximation is used here, which is well justified for the high-bias case. ${ }^{62,66}$ Indeed, under high bias, the electrons tunnel into the surface not only from the group of the last atoms at the tip apex, but from the mesoscopic surface of the tip. With the present model description the system is invariant with respect to translations parallel to the surface. The time-dependent wave function is then expressed as follows:

$$
\Psi(\mathbf{r}, t)=\frac{1}{2 \pi} e^{i \mathbf{k}_{\|} \cdot \mathbf{r}_{\|}} \psi(z, t),
$$

where $\mathbf{r}_{\|}=(x, y)$ and $\mathbf{k}_{\|}=\left(k_{x}, k_{y}\right)$ are the position and the electron wave vectors in the plane parallel to the surface, respectively. In what follows, we use the $z$ axis perpendicular to the surface of the overlayer with negative $z$ inside the $\mathrm{Pb} / \mathrm{Cu}(111)$ metallic structure, $z=0$ corresponds to the image plane of the overlayer defined in Sec. II B.

The time evolution of $\psi(z, t)$ is given by the time-dependent Schrödinger equation with Hamiltonian:

$$
\hat{H}=-\frac{1}{2} \frac{\partial^{2}}{\partial z^{2}}+\frac{k_{\|}^{2}}{2 m^{*}}+\mathbb{U}(z)+V_{\mathrm{abs}}(z)+V_{\mathrm{mb}}(z) .
$$

Within the present model, the energy dispersion of the states with $k_{\|}$is given by the free-electron parabola with effective electron mass $m^{*}=1$. The motion parallel to the surface can be factorized out, and it is sufficient to address numerically only the $\bar{\Gamma}$ case corresponding to $k_{\|}=0$.

$V_{\text {abs }}$ and $V_{\mathrm{mb}}$ in Eq. (2) are both non-Hermitian complex absorbing potentials, however with very different physical grounds. $V_{\mathrm{abs}}$ is introduced at the extremities of the $z$ mesh in order to avoid artificial effects because of the finite size of the computational box. ${ }^{70}$ It is thus a technical tool linked with the WPP. $V_{\mathrm{mb}}$ is active in the whole $\mathrm{Pb} / \mathrm{Cu}(111)$ sample and allows to account for the excited electron population decay due to many-body inelastic processes (see below). In this respect, it is similar to the complex absorbing potentials used in the simulations of low-energy electron diffraction. ${ }^{71}$

The wave function $\psi(z, t)$ is obtained numerically on the mesh of equidistant points in $z$. We use the short-time propagation: ${ }^{72} \psi(z, t+\Delta t)=\exp (-i \hat{H} \Delta t) \psi(z, t)$, subject to initial conditions $\psi(z, t=0)=\psi_{0}(z)$. The exponential of the Hamiltonian is calculated with split-operator technique, ${ }^{73}$ where the Fourier-grid approach ${ }^{74}$ is applied for the spatial derivatives entering the kinetic-energy operator.

Provided $\psi(z, t)$, the energies $\left(E_{n}\right)$ and lifetimes $\left(\gamma_{n}\right)$ of quasistationary QWSs (resonances) of $\mathrm{Pb} / \mathrm{Cu}(111)$ system are extracted from the Laplace transform $S(\omega)$ of the autocorrelation function $A(t):^{50}$

$$
\begin{aligned}
S(\omega) & =\int_{0}^{T} e^{i(\omega+i \eta) t} A(t) d t \\
& =\int_{0}^{T} d t e^{i(\omega+i \eta) t}\left[\int d z \psi_{0}^{*}(z) \psi(z, t)\right],
\end{aligned}
$$

To this end, the initial wave function $\psi_{0}(z)$ is typically chosen as a Gaussian wave packet located at the $\mathrm{Pb}$ /vacuum interface to ensure the overlap with QWSs characterized by different principal quantum numbers $n$. Once the energies $E_{n}$ are known, the wave functions of the QWSs can be obtained as:

$$
\psi_{n}(z)=\int_{0}^{T} e^{i\left(E_{n}+i \eta\right) t} \psi(z, t) d t .
$$

Comparison between calculations performed with and without biased STM tip sheds light on the role of the electric field in the STM junction.

Along with analysis of the electronic states of the system, the WPP allows one to calculate the transmission probability of the STM junction $T(E, V, Z)$ at $\bar{\Gamma}$ as a function of the electron energy $E$, applied bias $V$, and tip-surface distance $Z$. The initial wave function in this case is set as a combination of Gaussian wave packets incident from the tip onto the junction:

$$
\psi_{0}=\exp \left(-z^{2} / \Delta^{2}\right) \sum_{\ell=1}^{N} e^{-i k_{\ell} z}
$$

where $k_{\ell}$ are the momenta and $\Delta$ is the width of the incident wave packets. $N$ (typically two) is the number of wave packets necessary to ensure the required precision within the energy range of interest. The parameters $k_{\ell}$ and $\Delta$ are optimized for each bias. We sketch in Fig. 2 the probability density of an initial wave packet together with the system potential

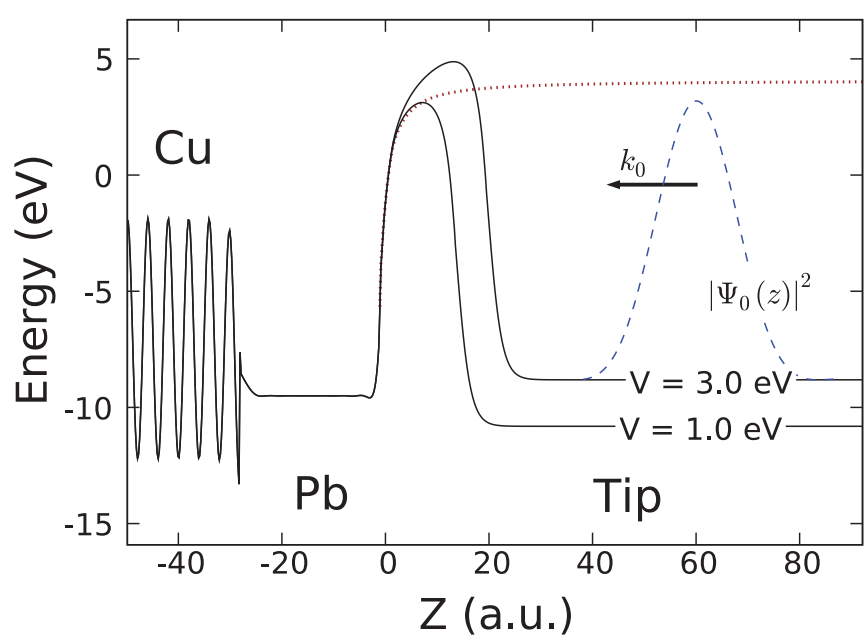

FIG. 2. (Color online) Black curves: Model potential of the tip $+5 \mathrm{ML}-\mathrm{Pb} / \mathrm{Cu}(111)$ system at two different bias values $V$ and the same current density. Energies are given with respect to the Fermi level. Red dotted curve: the potential of the bare $5 \mathrm{ML}-\mathrm{Pb} / \mathrm{Cu}(111)$ system. $z=0$ corresponds to the image plane position of $\mathrm{Pb}$ surface. The probability density of the Gaussian wave packet used in the WPP calculations is sketched with dashed curve. 
configuration for the particular coverage of $5 \mathrm{ML}$ and two different bias values.

Because of the $V_{\mathrm{mb}}$ term in Eq. (2), the flux is not conserved inside the $\mathrm{Pb} / \mathrm{Cu}(111)$ sample. The direct extraction of transmission from the flux analysis is not possible in this region. We use an alternative approach where, from the fluxconservation, $T(E, V, Z)=1-R(E, V, Z)$. The reflection coefficient $R(E, V, Z)$ is calculated with the virtual detector method $^{75}$ by placing the virtual detector in the asymptotic region inside the tip.

From the transmission probability one calculates the current density ( $0 \mathrm{~K}$ temperature) between the flat tip and the sample: ${ }^{76}$

$$
\begin{aligned}
I(V, Z)= & \frac{V}{2 \pi^{2}} \int_{-U_{0}+V}^{E_{F}} d E T(E, V, Z) \\
& +\frac{1}{2 \pi^{2}} \int_{E_{F}}^{E_{F}+V} d E\left(V+E_{F}-E\right) T(E, V, Z),
\end{aligned}
$$

where the lower limit $-U_{0}+V$ refers to the valence band bottom of the tip shifted by the applied bias, and $E_{F}$ corresponds to the Fermi level of the unperturbed system. From $I(V, Z)$ we obtain the current-voltage, conductance-voltage, or distance-voltage characteristics. The constant current distancevoltage characteristic $Z(V)$ is numerically differentiated to obtain the $d Z / d V$ spectra to be compared with experimental data. We also calculated the constant current $d I / d V$ spectra (not shown). The QWSs of the system appear as peak structures equally well resolved in both $d Z / d V$ spectra and differential conductance $d I / d V$. Indeed, $I=I(V, Z)$ and $Z=Z(V, I)$, so that the partial derivatives are related by $\left[\right.$ with $\left.(\partial I / \partial Z)_{V} \neq 0\right]$ :

$$
(\partial Z / \partial V)_{I}=-(\partial I / \partial Z)_{V}^{-1}(\partial I / \partial V)_{Z}
$$

The similarity between the two spectroscopies is a consequence of the fact that $(\partial I / \partial Z)_{V}$ is a smooth function of $V$,

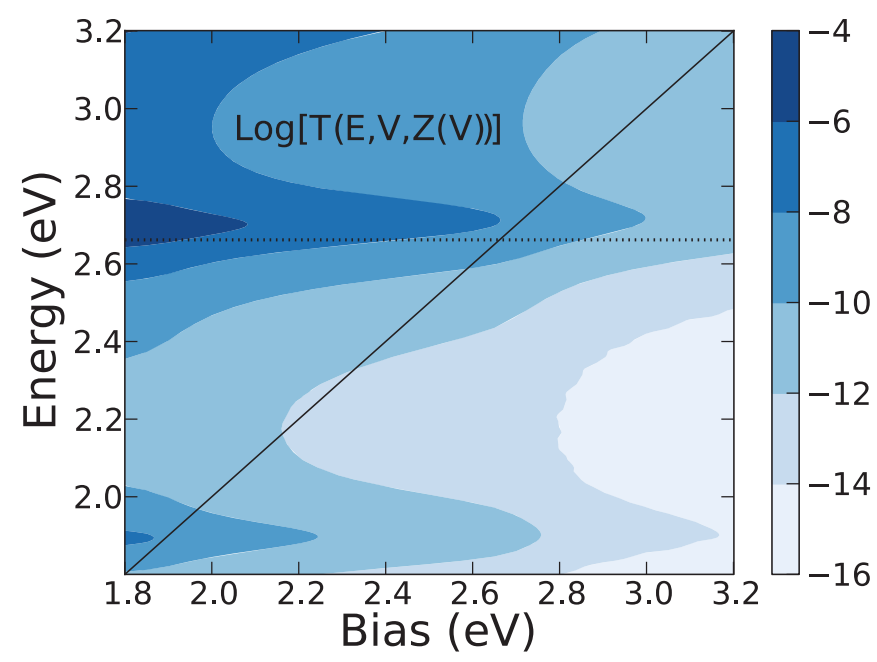

FIG. 3. (Color online) Calculated logarithmic transmission as a function of electron energy $E$ and applied bias $V$ for 15ML$\mathrm{Pb} / \mathrm{Cu}(111)$. The dotted line shows the energy of the $n=26$ QWS calculated for the bare system (no tip), where $n$ is a principal quantum number. The solid line $E=V$ defines a cut of the $T[E, V, Z(V)] 2 \mathrm{D}$ plots corresponding to the spectroscopic constant current mode (see Fig. 8). merely reflecting the evolution of the tunneling barrier between the tip and the surface.

The illustrative example of the calculated transmission probability $T[E, V, Z(V)]$ is shown in Fig. 3 for the 15-MLthick $\mathrm{Pb}$ overlayer on $\mathrm{Cu}(111)$. The results are presented as a function of the applied bias $V$, and electron energy $E$ measured with respect to the Fermi level. Note that the tip-surface distance $Z$ is bias-dependent because of the constant current mode, where the constant current density has been set as: $I=5 \times 10^{-2} \mathrm{nA} / \AA^{2}$. The transmission resonances observed in Fig. 3 correspond to the QWSs of the system. We would like to stress that the rigorous definition of these states assumes that the Hamiltonian is fixed, i.e., that the resonance analysis is performed for the constant bias $V$ cut of the 2D figure. For the fixed bias the transmission probability rises in overall with electron energy, reflecting decrease of the tunneling barrier. When bias is increased, the tip moves away from the surface to reestablish the constant current via overall reduction of the transmission probability. This is nicely seen in the figure when comparing data at the same energy, but for increasing bias. The solid line $E=V$ in the figure defines a cut of the $T[E, V, Z(V)] 2 \mathrm{D}$ plots corresponding to the spectroscopic constant current mode. Basically, the spectroscopically observed peaks are those of the $T[E=V, V, Z(V)]$ function.

\section{B. Model potential}

The $\mathrm{Pb} / \mathrm{Cu}(111)$ system is described with a $1 \mathrm{D}$ model potential derived from self-consistent calculations using densityfunctional theory within the local-density approximation (LDA). The potential only depends on the coordinate $z$ perpendicular to the surface. A free-electron motion parallel to the surface is assumed with effective mass $m^{*}=1$. This approach allows to account for both electronic structure and dynamical properties of $\mathrm{Pb}$ overlayers on $\mathrm{Cu}(111)$, as has been shown in previous works. ${ }^{37,48,50}$ The details on the potential construction can be found elsewhere. ${ }^{37,48,50} \mathrm{We}$ will only outline here the most important features specific for the present STS study.

The $\mathrm{Cu}(111)$ substrate is represented with the unscreened model potential proposed by Chulkov et $a l,{ }^{45}$ in which an atomic plane structure in $z$ direction is accounted for by a periodic function. The parameters of the potential are optimized in such a way that the experimental $\mathrm{Cu}(111)$ work function $(4.94 \mathrm{eV})$, as well as the projected band structure at the $\bar{\Gamma}$ point (see Fig. 1) are reproduced by the DFT-LDA calculation for pristine $\mathrm{Cu}(111)$ surface. The $\mathrm{Pb}$ overlayer is described by the stabilized jellium model where addition of a constant attractive potential inside the metal allows to retrieve the $\mathrm{Pb}$ work function value $(\Phi=4.08 \mathrm{eV})$ consistent with ab initio calculations ${ }^{35,42,77}$ and experimental data. ${ }^{46,78,79}$ The jellium description should hold in the energy range where the $p_{z}$ band of the parent bulk electronic structure along $\Gamma$-L can be approximated by a free-electron parabola (see Fig. 1). For each $\mathrm{Pb}$ overlayer thickness, the semi-infinite $\mathrm{Pb} / \mathrm{Cu}(111)$ potential is obtained by solving self-consistently the 1D Kohn-Sham equations within LDA.

The effective one-electron potential $V_{\text {eff }}$ obtained by the DFT-LDA scheme does not reproduce the long-range image 
charge interaction $V_{\mathrm{im}}$ for an electron being on the vacuum side above a metallic surface. $V_{\mathrm{im}}=\frac{-1}{4\left(z-z_{\mathrm{im}}\right)}$, where $z_{\mathrm{im}}$ is the position of the image plane, taken here as $1.23 a_{0}$ in front of the $\mathrm{Pb}$ jellium edge. ${ }^{80}$ The inclusion of the image potential tail is mandatory for the present study where we are interested in the QWSs with energies close to the vacuum level. Therefore the DFT potential has been corrected above the $\mathrm{Pb}$ overlayer as follows:

$$
V_{s}(z)= \begin{cases}\frac{A \exp \left[-\lambda\left(z-z_{0}\right)\right]-1}{4\left(z-z_{\mathrm{im}}\right)}, & z \geqslant z_{0} \\ V_{\mathrm{eff}}(z), & z<z_{0}\end{cases}
$$

where $V_{s}(z)$ is the electron-surface interaction used in the calculations and $z_{0}=z_{\text {im }}+0.5 a_{0}$. The rest of the parameters are fixed by the continuity of $V_{s}(z)$ and its derivative at $z_{0}$. Thus with $V_{s}(z)$ we can study the quantized states of the $\mathrm{Pb} / \mathrm{Cu}(111)$ system over a broad energy range, extending up to the vacuum level, where the mixing between the QWSs and the image potential states (ISs) becomes important.

The STM tip is represented with a 1D (flat-tip approximation) jellium model. The electron-tip interaction $V_{\text {tip }}(z)$ is given by Jones-Jennings-Jepsen potential: ${ }^{81}$

$$
V_{\text {tip }}(z)= \begin{cases}\frac{1-\exp \left[\lambda\left(z-z_{\text {tip }}\right)\right]}{4\left(z-z_{\text {tip }}\right)}, & z<z_{\text {tip }}, \\ \frac{U_{0}}{A \exp \left[-B\left(z-z_{\text {tip }}\right)\right]+1}, & z \geqslant z_{\text {tip }},\end{cases}
$$

where parameters $A$ and $B$ are fixed from continuity conditions at position of the image plane of the tip $z_{\text {tip }}{ }^{81}$ The potential inside the tip has been set to $U_{0}=-15.9 \mathrm{eV}(\mathrm{Al})$ and $\lambda=$ $0.9 a_{0}^{-1}$ has been used. We checked that results do not change with $U_{0}=-13.55 \mathrm{eV}$ (as calculated with present DFT for $\mathrm{Pb}$ ). Indeed, in the tunneling regime, the transmission resonances are determined by the energies and lifetimes of the QWSs of the $\mathrm{Pb}$ overlayer on $\mathrm{Cu}(111)$. As far as the tip does not present resonant features in the electronic density of states (DOS), the tip properties have little impact on the resonance tunneling profile.

For an electron between two infinite conductor plates $\left(z_{\text {im }}<\right.$ $\left.z<z_{\text {tip }}\right)$, the potential due to the multiple electrostatic images, and due to the applied bias reads: ${ }^{62}$

$$
\begin{aligned}
V_{\mathrm{mi}}(z)= & \frac{1}{4} \sum_{n=1}^{\infty}\left\{\frac{-1}{\left(z-z_{\mathrm{im}}\right)+n Z}\right. \\
& \left.+\frac{-1}{\left(z-z_{\text {tip }}\right)+n Z}+\frac{2}{n Z}\right\}+\left(z-z_{\mathrm{im}}\right) V / Z,
\end{aligned}
$$

where the tip- $\mathrm{Pb} / \mathrm{Cu}(111)$ surface distance is defined as $Z=$ $z_{\text {tip }}-z_{\text {im }}$.

Finally, the total effective potential acting on the tunneling electron is given by:

$$
V_{\Sigma}(z)= \begin{cases}V_{s}(z), & z \leqslant z_{\text {im }}, \\ V_{s}(z)+V_{\text {tip }}(z)+V_{\text {mi }}(z), & z_{\text {im }}<z<z_{\text {tip }}, \\ V_{\text {tip }}(z)+V, & z \geqslant z_{\text {tip }} .\end{cases}
$$

Depending on the problem at hand, the WPP calculations were performed with potential $\mathbb{U}(z)$ [see Eq. (2)] given by all or some terms entering Eq. (11). Thus, in the search of the reference QWSs of the unperturbed $\mathrm{Pb} / \mathrm{Cu}(111)$ system, $\mathbb{U}(z)=V_{s}(z)$ has been used. The evolution of the QWSs under the applied electric field in the junction, $\mathcal{E}=V / Z$, has been studied with

$$
\mathbb{U}(z)=V_{s}(z)+\left(z-z_{\mathrm{im}}\right) \mathcal{E} \theta\left(z-z_{\mathrm{im}}\right),
$$

where $\theta(z)$ is the Heaviside step function. Finally, the transmission of the junction has been calculated with the full potential: $\mathbb{U}(z)=V_{\Sigma}(z)$.

Figure 2 shows the total potential $V_{\Sigma}$ for a system comprising $5 \mathrm{ML}$ of $\mathrm{Pb}$ on $\mathrm{Cu}(111)$ and the STM tip placed at distance $Z=11.5$ (17.6) $a_{0}$ from the image plane position at bias $V=1.0(3.0) \mathrm{eV}$.

\section{Many-body contribution}

The WPP is a one-electron approach so that for the system described with the Hermitian part of the potential $\mathbb{U}(z)$, only the elastic population decay of the QWSs via resonant electron tunneling into the $\mathrm{Cu}(111)$ bulk is accounted for. The QWSs are stationary within the one-electron picture if their energies $E_{n}$ fall into the projected band gap of $\mathrm{Cu}(111)$ at $\bar{\Gamma}:-5.1<$ $E<0.04 \mathrm{eV}$ with respect to the vacuum level $(-1.02<E<$ $4.12 \mathrm{eV}$ with respect to the Fermi level). Outside this range, the electron tunneling into the substrate is possible and the QWSs become quasistationary (resonances). Their width is given by the one-electron decay rate into the substrate, $\gamma_{1 e}$.

The excited-state population decay via inelastic many-body processes can be included in the WPP scheme through the non-Hermitian term(s) in the Hamiltonian. ${ }^{69}$ We use a local formulation of the complex many-body potential $V_{\mathrm{mb}}$ :

$$
V_{\mathrm{mb}}=\frac{-i}{2}\left[\gamma_{e-\mathrm{ph}}+\gamma_{e-e}\right] \frac{1}{1+\exp \left[\left(z-z_{\mathrm{jel}}\right) / \delta\right]},
$$

The parameter of the switching function is $\delta=0.09 a_{0} \cdot \gamma_{e-\mathrm{ph}}$ stands for the contribution of the electron-phonon scattering to the population decay and $\gamma_{e-e}$ stands for the contribution of the electron-electron scattering. $\gamma_{e-p h}$ and $\gamma_{e-e}$ are fixed on the basis of the earlier calculations of the inelastic broadening of the QWSs in the $\mathrm{Pb} / \mathrm{Cu}(111)$ system. Indeed, the electronic wave functions $\psi_{n}$ of the QWSs with energies in the projected band gap of the substrate well below the vacuum level are localized inside the $\mathrm{Pb}$ overlayer. The perturbation estimate for the many-body decay rates $\gamma_{\mathrm{mb}}$ gives: $-i \gamma_{\mathrm{mb}} / 2=\left\langle\psi_{n}\left|V_{\mathrm{mb}}\right| \psi_{n}\right\rangle$, where from Eq. (13) it follows that $\gamma_{\mathrm{mb}} \approx \gamma_{e-\mathrm{ph}}+\gamma_{e-e}$. Thus the above choice of parameters guarantees that, in the absence of the STM tip, the inelastic broadening $\gamma_{\mathrm{mb}}$ obtained in WPP calculations reproduces the earlier results reported for the QWSs, as we have checked with our calculations.

Specifically, for the inelastic electron-phonon decay $\gamma_{e-\mathrm{ph}}=20 \mathrm{meV}$ has been used. This value corresponds to the averaged $\gamma_{e-\mathrm{ph}}$ at $5 \mathrm{~K}$ calculated for the QWSs in free-standing Pb slabs. ${ }^{41}$ The inelastic electron-electron decay $\gamma_{e-e}$ requires more involved treatment. In our previous work, ${ }^{50}$ we found that for low-lying QWSs close to the Fermi level, the dependence of $\gamma_{e-e}$ on the energy of the QWS is described with the 3D Fermi-liquid dependence given by the Quinn-Ferrel (QF) formula. ${ }^{82}$ Above $1 \mathrm{eV}$ with respect to the Fermi level, $\gamma_{e-e}$ changes quasilinearly with QWS energy. The absolute values for the decay rates agree with TR-2PPE studies. ${ }^{23}$ The trend for the linear dependence of the QWSs linewidths with energy was recently reported in an STS study of $\mathrm{Pb} / \mathrm{Ag}(111){ }^{22}$ Consistently with above findings, we model the electron- 
electron contribution to the excited-state decay as

$$
\gamma_{e-e}= \begin{cases}2.5019 r_{s}^{5 / 2}\left(E-E_{F}\right)^{2}, & E-E_{F} \leqslant 1 \mathrm{eV}, \\ \alpha\left(E-E_{F}\right)+\beta, & E-E_{F}>1 \mathrm{eV},\end{cases}
$$

where the screening radius $r_{s}=2.3 a_{0} \quad(\mathrm{~Pb}), \quad \alpha=$ $52 \mathrm{meV} \mathrm{eV}^{-1}$, and $\beta=-41 \mathrm{meV}$. We have checked that changing the absorption in $\mathrm{Cu}$ substrate does not affect the final results. The main broadening of the QWSs thus comes from the inelastic scattering in $\mathrm{Pb}$ overlayer.

The energy dependence of the $\gamma_{e-e}$ implies that, in principle, for each energy, the transmission $T(E, V, Z)$ at $\bar{\Gamma}$ should be calculated with the corresponding $\gamma_{e-e}$. This approach is extremely time consuming and would be out of reach. However, we have checked that using $\gamma_{e-e}$ at the energy given by the applied bias $E=V+E_{F}$ does change the energy dependence of the transmission but does not affect the calculated distance-voltage $d Z / d V$ characteristic. This is because the $d Z / d V$ spectroscopy is mainly sensitive to the narrow energy interval close to the tip Fermi level where the electrons injected into $\mathrm{Pb} / \mathrm{Cu}(111)$ have energy $E \simeq V+E_{F}$.

Observe that for an electron injected from the STM tip into the surface along $\Gamma$-L, the one-electron reflectivity of the $\mathrm{Pb} / \mathrm{Cu}(111)$ interface is one within the $\mathrm{Cu}(111)$ projected band gap. With the flat-tip approximation, the tunneling flux through the junction in this case is only possible due to the inelastic energy relaxation processes described by $V_{\mathrm{mb}}$.

\section{RESULTS AND DISCUSSION}

\section{A. Effect of the tip: Stark shift}

We start our discussion with the effect of the electric field in the STM junction on the QWSs. In Fig. 4, we show the

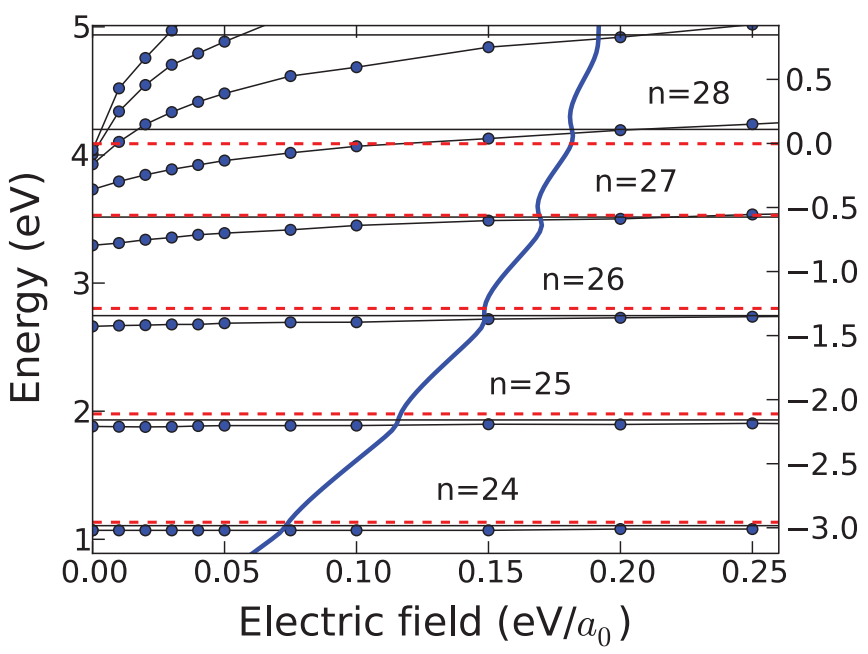

FIG. 4. (Color online) Applied-field dependence of the energies of the QWSs. Solid lines with dots: calculated energies of the QWSs as a function of the applied uniform electric field $\mathcal{E}$ for the system comprising the STM tip $+15 \mathrm{ML}-\mathrm{Pb} / \mathrm{Cu}(111)$ system. Energies are given with respect to the Fermi (vacuum) level as indicated in the left (right) axis. Horizontal dashed lines: energies of the QWS peaks in STS experiments. ${ }^{21}$ Horizontal solid lines: energies of the QWS peaks in the calculated $d Z / d V$ spectra. The blue curve represents the electric field as calculated at each bias (energy with respect to the Fermi level) for the constant current spectroscopy. calculated energies $E_{n}(\mathcal{E})$ of the QWSs as a function of the applied field in the STM junction $\mathcal{E}=V / Z$. Here, the WPP method with a potential given by Eq. (12) has been used. Results are shown within the energy range of $1<E<5 \mathrm{eV}$ with respect to the Fermi level, i.e., the one encompassed in the constant current STS experiments. ${ }^{21,22}$ The principal quantum number $n$ of the states, as indicated in the figure, corresponds to the nodal structure of the wave function. ${ }^{50}$

At zero field, the QWSs mix with image potential states (ISs) forming Rydberg-like series converging toward the vacuum level. The mixed-character of these QWS-ISs has been discussed earlier for the $\mathrm{Au} / \mathrm{Pd}(111)$ system. ${ }^{83}$ It can be easily understood in terms of the phase accumulation model. ${ }^{24}$ The QWSs are associated with electronic standing waves due to confinement between the film/substrate and film/vacuum interfaces. The quantization condition is

$$
2 \pi n=2 k\left(E_{n}\right) D+\varphi_{\mathrm{Cu}}\left(E_{n}\right)+\varphi_{\mathrm{vac}}\left(E_{n}\right),
$$

where $n$ is a quantum number, $k(E)$ is the bulk $\mathrm{Pb}$ band dispersion in the direction perpendicular to the overlayer, $D$ is the overlayer thickness, and $\varphi_{\mathrm{vac}}\left(\varphi_{\mathrm{Cu}}\right)$ is the phase shift due to the scattering at vacuum (substrate) interface. For $E_{n}$ approaching the vacuum level $E_{V}$, the $\varphi_{\mathrm{vac}}$ is given by ${ }^{25}$

$$
\varphi_{\mathrm{vac}}\left(E_{n}\right)+\pi=\pi / \sqrt{8\left(E_{V}-E_{n}\right)} .
$$

Since $\varphi_{\text {vac }}$ diverges for $E_{n} \rightarrow E_{V}$, it follows from Eq. (15) that an infinite series of states is formed. The $\varphi_{\text {vac }}$ phase variation with energy is much faster than that for the phase accumulated inside the layer. Then, each new state within a narrow energy range close to the vacuum level corresponds to an additional zero in the wave function appearing in the vacuum side. It is exactly what one has for the ISs described by Eq. (15) with $D=0$ and forming the Rydberg-like series with $E_{n^{\prime}}=$ $1 / 32\left(n^{\prime}+a\right)^{2}$. Here, $a$ is the quantum defect, and quantum number $n^{\prime}$ reflects the nodal structure of the IS wave function in the vacuum region. ${ }^{24,57,58}$ The mixed QWS-IS character of the states close to the vacuum level is reflected in their electronic densities. Appreciable part of the electron density is moved from inside the $\mathrm{Pb}$ film into the vacuum, as seen in Fig. 5 where we show the wave functions $\psi_{n}$ of the states obtained from WPP. From the nodal structure in vacuum, one can infer the principal quantum number $n^{\prime}$ of the IS providing the leading contribution to $\psi_{n}$ (indicated in parenthesis in the figure).

When the bias (electric field) is applied to the junction, the low-energy QWSs localized mainly inside the $\mathrm{Pb}$ layer experience only minor energy shift in agreement with experimental results. ${ }^{84,85}$ At variance, the states with mixed IS character close to the vacuum level are very sensitive to the applied electric field because of the high probability of the electron presence above the surface of the film. These states experience appreciable energy shift (Stark shift), so that the Rydberg-like series converging toward the vacuum level is destroyed already for fields as low as $0.05 \mathrm{eV} / a_{0}$. Note that the field $\mathcal{E}=0.05 \mathrm{eV} / a_{0}$ corresponds to a tip-surface distance as large as $4 \mathrm{~nm}$ for a bias of $4 \mathrm{eV}$. The actual electric fields obtained with the WPP for the conditions of constant current STS experiments (see below) of Yang et al. ${ }^{21}$ are also shown in Fig. 4. The field dependence of the Stark shift of the states with 

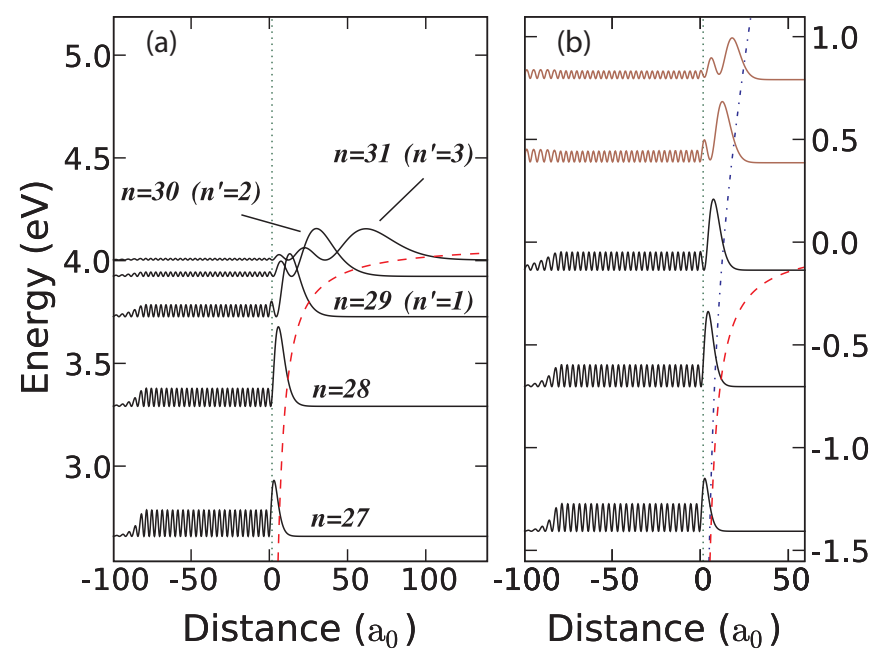

FIG. 5. (Color online) Change of the wave functions of the QWSs in the $15 \mathrm{ML}-\mathrm{Pb} / \mathrm{Cu}(111)$ system under applied electric field. Solid lines represent electronic densities of the QWSs. The principal quantum number $n$ is indicated above each state. The image plane position is denoted by a vertical dotted line. Energies are given with respect to the Fermi (vacuum) level labeled at the left (right) axis. (a) Zero field case. The number in parenthesis indicates the $n^{\prime}$ (the quantum number of the IS with leading contribution to the mixed QWS-IS state). The image potential at the vacuum side of the $\mathrm{Pb}$ film is shown by dashed line. (b) The case of the uniform electric field of the strength $\mathcal{E}=0.05 \mathrm{eV} / a_{0}$. The densities of the quasistationary states with energies above the $\mathrm{Cu}(111)$ projected band gap, and therefore decaying via one-electron transfer into the substrate, are represented with red color. Dashed line: image potential at the vacuum side of the $\mathrm{Pb}$ film, dashed dotted line: total (image + linear ramp) potential.

mixed QWS-IS character calculated here is very close to the ab initio results ${ }^{64}$ as well as the experimental data supported with model calculations ${ }^{59}$ for the ISs.

Thus the electric field in the junction overrides the image potential. For the pristine metal surfaces, the ISs evolve into the field emission resonances (FERs) with energy quantization given by ${ }^{59-66} E_{n^{\prime}}=\frac{1}{2}\left[3 \pi \mathcal{E}\left(n^{\prime}-1 / 4\right)\right]^{2 / 3}\left(n^{\prime}=1,2, \ldots\right)$. Similarly, for the $\mathrm{Pb}$ overlayer, the states with mixed QWSIS character evolve into the states with mixed QWS-FER character, as reflected in their wave functions. The WPP results for the wave functions of the QWS in biased STM junction are shown in Fig. 5 for the electric field $\mathcal{E}=0.05 \mathrm{eV} / a_{0}$. Compared to the bare $\mathrm{Pb} / \mathrm{Cu}(111)$ case, the electronic densities of the high-energy states are pushed by the constant electric field toward the surface. However, partial electron spread from the $\mathrm{Pb}$ film into vacuum is preserved. The same features have been reported for the Stark-shifted ISs. ${ }^{86}$

The Stark shift of the ISs [which is as strong as $0.3 \mathrm{eV}$ for $n^{\prime}=1$ IS at clean $\mathrm{Cu}(111)$ surface ${ }^{65}$ ] shows that for the STM junction under the bias, the phase accumulation model with vacuum scattering phase described with Eq. (16) can not provide an adequate description of the system. The scattering phase shift $\varphi_{\text {vac }}$ at the $\mathrm{Pb}$ vacuum interface is rather that of the triangular potential barrier, ${ }^{61,62,87}$

$$
\varphi_{\mathrm{vac}}+\pi / 2=2\left[2\left(E_{n}-E_{V}\right)\right]^{3 / 2} / 3 \mathcal{E},
$$

as can be obtained from quasiclassical theory or from the asymptotic properties of the Airy functions. ${ }^{88}$ We would like to emphasize here that the present WPP calculations are performed with the complete potential given by Eq. (11), and so are free from any approximative description of $\varphi_{\mathrm{vac}}$.

To summarize, the STS experiment does not appear to be noninvasive, as far as the QWSs close to the vacuum level are concerned. Because of the electric field in the STM junction, the energy of these states is raised by the Stark shift. As a result, the QWSs with mixed IS character transform into QWSs with mixed FER character. Both, the energy upshift and change in the wave function would increase the rate of the inelastic decay of the states, ${ }^{86}$ and so the linewidth of the structures in $d Z / d V$ and $d I / d V$ spectra. Note that above $4.1 \mathrm{eV}$ with respect to the Fermi level (above $\mathrm{Cu}$ L gap), the QWSs are in resonance with propagating electronic states of the $\mathrm{Cu}(111)$ substrate. The resonant electron transfer into the substrate constitutes then an additional channel of the QWSs population decay.

\section{B. STS spectra}

We now turn to the calculated STS spectra and their comparison with experimental data. The constant current $d Z / d V$ curves were calculated for overlayer thicknesses ranging from 4 to $18 \mathrm{ML}$. The current density was kept constant for all calculations, and it was set to $I=5 \times 10^{-2} \mathrm{nA} / \AA^{2}$, such that the experimentally measured energy $(3.52 \mathrm{eV})$ of the $n=27$ peak is well reproduced for the $15-\mathrm{ML} \mathrm{Pb}$ film case. This implies the tip-surface distance of $Z=22 a_{0}$ at the bias $V=3.52 \mathrm{eV}$. If the effective radius of the tip $R_{\text {eff }}=20 \AA$ is assumed, the corresponding current through the STM junction is $\mathcal{I}=\pi R_{\text {eff }}^{2} I \approx 63 \mathrm{nA}$. The above choice of parameters appears quite reasonable when comparing with existing approaches. ${ }^{62,66}$

The $d Z / d V$ curves obtained for the $\mathrm{Pb}$ coverages of $10 \mathrm{ML}$ and $17 \mathrm{ML}$ are displayed in Fig. 6 together with experimental STS spectra of Ref. 21. The QWS energies calculated for

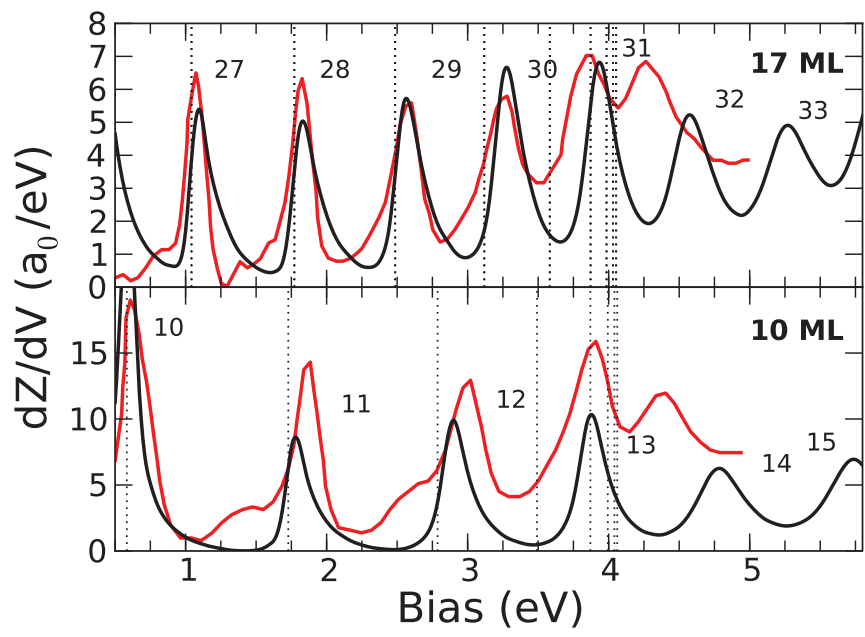

FIG. 6. (Color online) Comparison between calculated $d Z / d V$ curves (black) and experimental spectra (red) by Yang et al. ${ }^{21}$ Experimental spectra are rescaled for the sake of comparison. For further details see the main text. The calculated QWS energies of the bare $\mathrm{Pb} / \mathrm{Cu}(111)$ system are denoted by vertical dotted lines. 
the bare $\mathrm{Pb} / \mathrm{Cu}(111)$ without tip are also shown in the figure with vertical dashed lines. We use the labeling $n$ of the spectral peaks according to the principal quantum number of the underlying QWSs obtained from the calculated nodal structure of their wave functions. Note that in Ref. 21 the labels $n_{\text {exp }}$ differ from the present definition of $n$ as $n_{\exp }=n-1$. However, $n_{\exp }$ were not directly obtained but were deduced from the modeling of the data with the phase accumulation model. Furthermore, we refer to the thickness of the $\mathrm{Pb}$ film with respect to the $\mathrm{Cu}(111)$ surface, while in Ref. 21 the thickness is referred with respect to the $\mathrm{Pb}$ wetting layer. Thus, e.g., the present 17-ML case has to be compared to the 16-ML result as reported in the experimental work.

With the above remarks, the calculated STS spectra are in good agreement with experimental data for the energies below $4 \mathrm{eV}$ with respect to the Fermi level of the surface. The flat-tip approximation used here allows to reproduce experimental energy positions of the resonant features as well as their widths, including the overall peak broadening with increasing energy. At high energy (bias) above $4.12 \mathrm{eV}$, i.e., above the $\mathrm{L}$ gap of $\mathrm{Cu}(111)$, the calculated peaks broaden and loose their intensity. This is linked with opening of the QWSs decay channel via resonant one-electron tunneling into the $\mathrm{Cu}(111)$ substrate. At the same time, the regular energy progression of the calculated peaks is maintained, in contrast to experimental data. The difference between the theoretical and experimental data in the 4-5 eV energy range is directly linked to the $\mathrm{Pb}$ band structure, as will be discussed in detail in Sec. D.

The good agreement between theory and experiment for $V<4 \mathrm{eV}$ allows us to discuss some issues linked with the tunneling spectroscopy studies of the QWSs.

First, the difference between the energies of the resonant structures in the calculated $d Z / d V$ spectra $E_{n}^{\text {STS }}$ and the energies of the QWSs of the bare $\mathrm{Pb} / \mathrm{Cu}(111)$ system $E_{n}$ sets the energy shift $\Delta^{\text {STS }}$ inherent to the experimental procedure as:

$$
\Delta_{n}^{\mathrm{STS}}=E_{n}^{\mathrm{STS}}-E_{n} .
$$

$\Delta_{n}^{\mathrm{STS}}$ is shown in Fig. 7(a) for the case of the 17-ML Pb film. In full agreement with the discussion presented in the previous section (see Fig. 4), the measured peak positions reflect the energies of the QWSs of bare $\mathrm{Pb} / \mathrm{Cu}(111)$ only for the bias below $\approx 2 \mathrm{eV}$, where $\Delta_{n}^{\mathrm{STS}}$ is small. At higher bias the Stark shift modifies the energies of the QWSs. This effect becomes particularly important for the QWSs with mixed ISs character close to the vacuum level of the $\mathrm{Pb}$ overlayer. Thus the spectroscopic feature at the vacuum level $(4 \mathrm{eV})$ is associated with the Stark shift of the underlying QWS by $0.4 \mathrm{eV}$, which is of the same order of magnitude as reported for the first IS, at $\mathrm{Cu}(111){ }^{65}$

Second, the peaks in the STS spectra show an additional broadening of $0.2 \mathrm{eV}$, as compared to the decay rates of the underlying QWSs resonances. A similar result has been reported in the studies of the FERs. ${ }^{66}$ The broadening of the spectroscopic features is illustrated in Fig. 8. The calculated energy-dependent transmission $T[E, V, Z(V)]$, dynamic transmission $T[V, V, Z(V)]$, and $d Z / d V$ spectrum are presented for the 17-ML $\mathrm{Pb}$ coverage case. We recall that $Z(V)$ is defined by the constant current condition. The figure spans the energy range of the $n=29$ QWS peak (as appears in Fig. 6). The
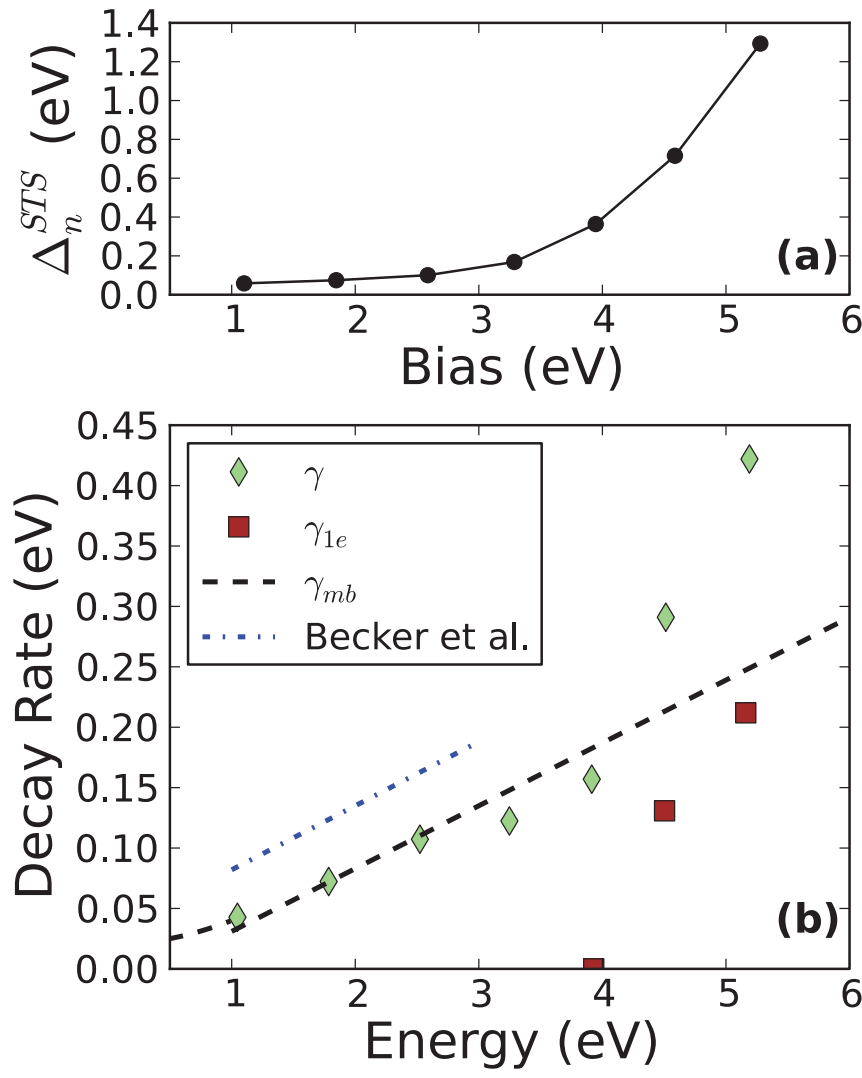

FIG. 7. (Color online) (a) Energy shift $\Delta_{n}^{\text {STS }}$ of the QWS peaks in the calculated $d Z / d V$ spectra. $\Delta_{n}^{\text {STS }}$ is defined with respect to the energies of the QWSs in the bare $17 \mathrm{ML}-\mathrm{Pb} / \mathrm{Cu}(111)$ system. (b) Calculated decay rates $\gamma_{n}$ of the QWSs for the same system, as a function of their energy $E_{n}$ with respect to Fermi level. $\gamma_{n}$ is obtained for the fixed bias $V$ and position of the tip $Z(V)$, where $E_{n}=V$. We also plot the many-body decay rate $\gamma_{e-\mathrm{ph}}+\gamma_{e-e}$ for the excited states inside the overlayer. The dashed-dotted line represents the linear fit to the QWS linewidths as deduced from experimental data in Ref. 22.

$T[E, V, Z(V)]$ has a well-defined Lorentzian profile centered at resonance energy and width $\gamma_{n}$ corresponding to the inverse lifetime of the $n=29$ QWS resonance. There is only a minor energy shift and nearly no width change for the bias variations within the resonance. The bias is varied by steps of $0.05 \mathrm{eV}$, and the precise $V$ value corresponding to each curve is set by the open dot located at $E=V$. Similar to the transmission, the dynamic transmission given by the thick line connecting $E=V$ points also shows quite a symmetric profile with no additional broadening. At variance, the calculated peak in $d Z / d V$ is asymmetric with a width $\gamma_{n}^{\prime} \approx \gamma_{n}+0.2 \mathrm{eV}$, and a maximum position shifted by $\sim 30 \mathrm{meV}$. This small energy shift appears nearly independent on the QWS under study. It is at the origin of the nonzero onset of $\Delta_{n}^{\text {STS }}$ for low bias, as seen in Fig. 7.

Because of the asymmetric structure of the measured and calculated STS peaks as well as because of the peak broadening inherent to the STS at high bias, the extraction of the corresponding decay rates is not a trivial task. We have not attempted any transformation of the experimental data of Yang et al. ${ }^{21}$ The WPP allows a direct extraction of the decay rates $\gamma_{n}$ from the calculations performed for the fixed 


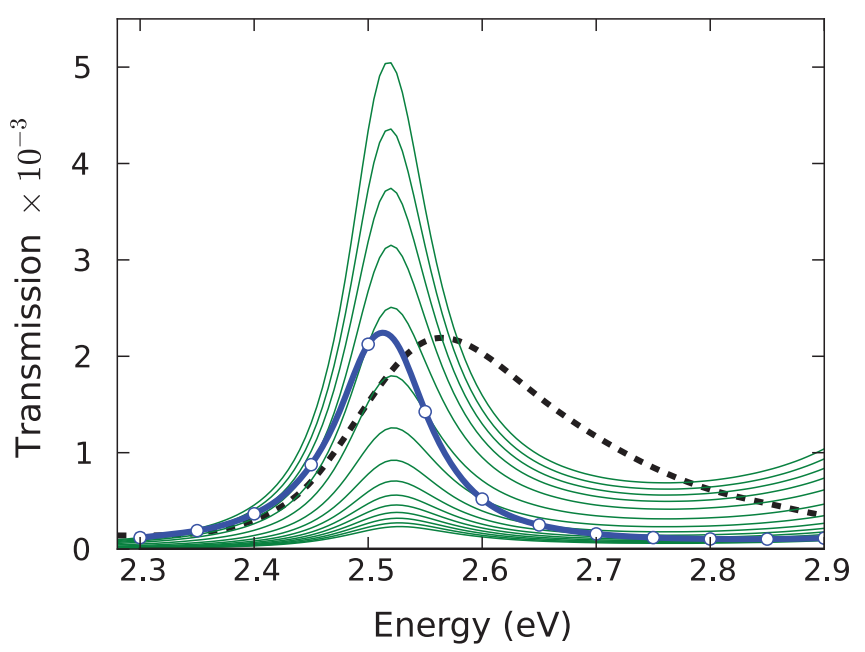

FIG. 8. (Color online) The transmission $T[E, V, Z(V)]$, dynamic transmission $T[V, V, Z(V)]$, and $d Z / d V$ spectrum for the $n=$ 29 QWS peak of the $17 \mathrm{ML}-\mathrm{Pb} / \mathrm{Cu}(111)$ system. The calculated transmission (thin lines) is shown as a function of the electron energy for different bias values. The $V$ value corresponding the each curve is set by the open dot located at $E=V$. The dynamic transmission is given by the thick line connecting the $E=V$ points. The $d Z / d V$ curve (dashed line) has been renormalized for the sake of comparison.

bias $V=E_{n}$ and fixed position of the tip $Z(V)$. Thus, the Hamiltonian is fixed so that the quasistationary states of the system can be rigorously defined. Here $E_{n}$ is the energy of the quasistationary QWS under study. We note that, a priori, $\gamma_{n}$ comprises several contributions: $\gamma_{n}=\gamma_{\mathrm{mb}}+\gamma_{1 e}+\gamma_{\mathrm{tip}} \cdot \gamma_{\mathrm{mb}}$ stands for the inelastic many-body decay, $\gamma_{1 e}$ is the rate of the elastic one-electron tunneling into the $\mathrm{Cu}(111)$ substrate for the energies above the $\mathrm{L}$ gap of $\mathrm{Cu}(111)$ at $\bar{\Gamma}$ (i.e., above $4.12 \mathrm{eV}$ ), and finally, $\gamma_{\text {tip }}$ is the rate of the elastic one-electron tunneling into the STM tip. Since in the present conditions the tip-surface distances are such that the transmission is in the $10^{-4}$ range, the contribution of the latter decay channel can be safely neglected.

In Fig. 7, we show the WPP results for the case of the 17-ML $\mathrm{Pb}$ film together with the decay rates extracted in Ref. 22 from the experimental study of $\mathrm{Pb} / \mathrm{Ag}(111)$. The difference in the substrate affects the $\gamma_{1 e}$. However, for the QWSs inside the projected band gap $\gamma_{1 e}=0$, and $\gamma_{\mathrm{mb}}$ is determined mainly by the inelastic hot-electron decay in the $\mathrm{Pb}$ overlayer as we have checked with WPP calculations by varying the absorbing potential inside the $\mathrm{Cu}(111)$ substrate. Thus the comparison with experimental data of Ref. 22 is meaningful. The present theoretical results can be well understood on the basis of the wave-function penetration arguments often involved in the discussion of the lifetimes of the ISs at surfaces. ${ }^{24,69}$ At low energies, the wave functions of the QWSs are confined within the $\mathrm{Pb}$ overlayer (see Fig. 5). The $\gamma_{\mathrm{mb}}$ decay rate is then given by the sum of the electron-electron and electronphonon contributions $\gamma_{\mathrm{mb}}=\gamma_{e-\mathrm{ph}}+\gamma_{e-e}$ to the excited-state population decay in the $\mathrm{Pb}$ film. It is directly included in the many-body absorbing potential (see Sec. II C), and represented by the dashed line in Fig. 7. For the energy range between 3 and $4 \mathrm{eV}$, the QWSs mix with FERs. Part of the electron density is moved into the vacuum, reducing the probability of interaction with possible excitations. As a result, the many-body decay rate of the corresponding states becomes smaller than that inside the $\mathrm{Pb}$ film. For the energies above $4.12 \mathrm{eV}$, the one-electron decay channel into the $\mathrm{Cu}(111)$ bulk opens. The $\gamma_{1 e}$ decay rate rapidly increases when energy rises above the band-gap threshold. The QWSs acquire an additional broadening, and the total width of the resonances is brought into the $0.3-0.4 \mathrm{eV}$ range.

\section{STS results for different $\mathrm{Pb}$ coverages}

Figure 9 summarizes our results on the STS of the $\mathrm{Pb} / \mathrm{Cu}(111)$ system. The energies of the calculated QWS peaks in the $d Z / d V$ spectra are presented as a function of overlayer thickness for $\mathrm{Pb}$ coverages ranging from 4 to $18 \mathrm{ML}$. Theoretical data are compared with STS experiments. ${ }^{21}$ As a reference, we also show the calculated energies of the QWSs in the bare $\mathrm{Pb} / \mathrm{Cu}(111)$ system (no tip-induced perturbation) as well as the QWSs energies obtained from the two-photon photoemission (2PPE) experiments. ${ }^{23}$

Our calculations are in agreement with available experimental data for the energies below $\sim 4 \mathrm{eV}$. The correspondence between the calculated and measured STS peak positions is particularly remarkable for coverages above $11 \mathrm{ML}$. When comparing the STS data with the QWSs energies of the bare $\mathrm{Pb} / \mathrm{Cu}(111)$ system, the importance of the Stark shift due to the applied bias, as discussed in the previous subsection, is evident for the high energy states. We stress here that this effect has not received enough attention in the previous analysis of the experimental STS data on Pb overlayers. ${ }^{21,22}$

At low coverages the calculated spectroscopic peak positions are systematically below the experimental data. This can not be the deficiency of the present modeling of the STM junction since all the QWSs, including those close to the Fermi level, are concerned. Indeed, the low-energy QWSs are well-localized inside the overlayer and thus are not sensitive to the potential above the $\mathrm{Pb}$ surface. We tentatively attribute the difference between calculated and measured data to the present DFT-LDA modeling of the bare $\mathrm{Pb} / \mathrm{Cu}(111)$ system. It results in the underestimation of the energies of the QWSs at low coverages as has been discussed in an earlier publication. ${ }^{48}$ The effects such as the specificity of the wetting layer as compared to the next layers and oscillations both in work function and $\mathrm{Pb}$ interlayer spacings with overlayer thickness will determine the precise value of the QWS energies. In fact, the good agreement between theoretical and experimental data above 11 ML coincides with coverages having converged work function, i.e., that of $\mathrm{Pb}(111)$ within $0.05 \mathrm{eV}$ (see also Ref. 35 and Ref. 79).

\section{Effect of the realistic band dispersion of $\mathrm{Pb}(111)$ along $\Gamma-\mathrm{L}$}

As follows from the results reported in Fig. 9 (see also Fig. 6), above $4 \mathrm{eV}$, the theoretically calculated peaks in the STS spectra, and thus, the underlying QWSs, continue regular progression towards higher energies $E_{n}$ with increasing principal quantum number. In contrast, the experimental data show the energy separation $E_{n+1}-E_{n}$ decreasing with $n$ within this energy range. Finally, measured QWSs peaks level off at $4.6 \mathrm{eV}$ energy. In some way, the energy dispersion of the 


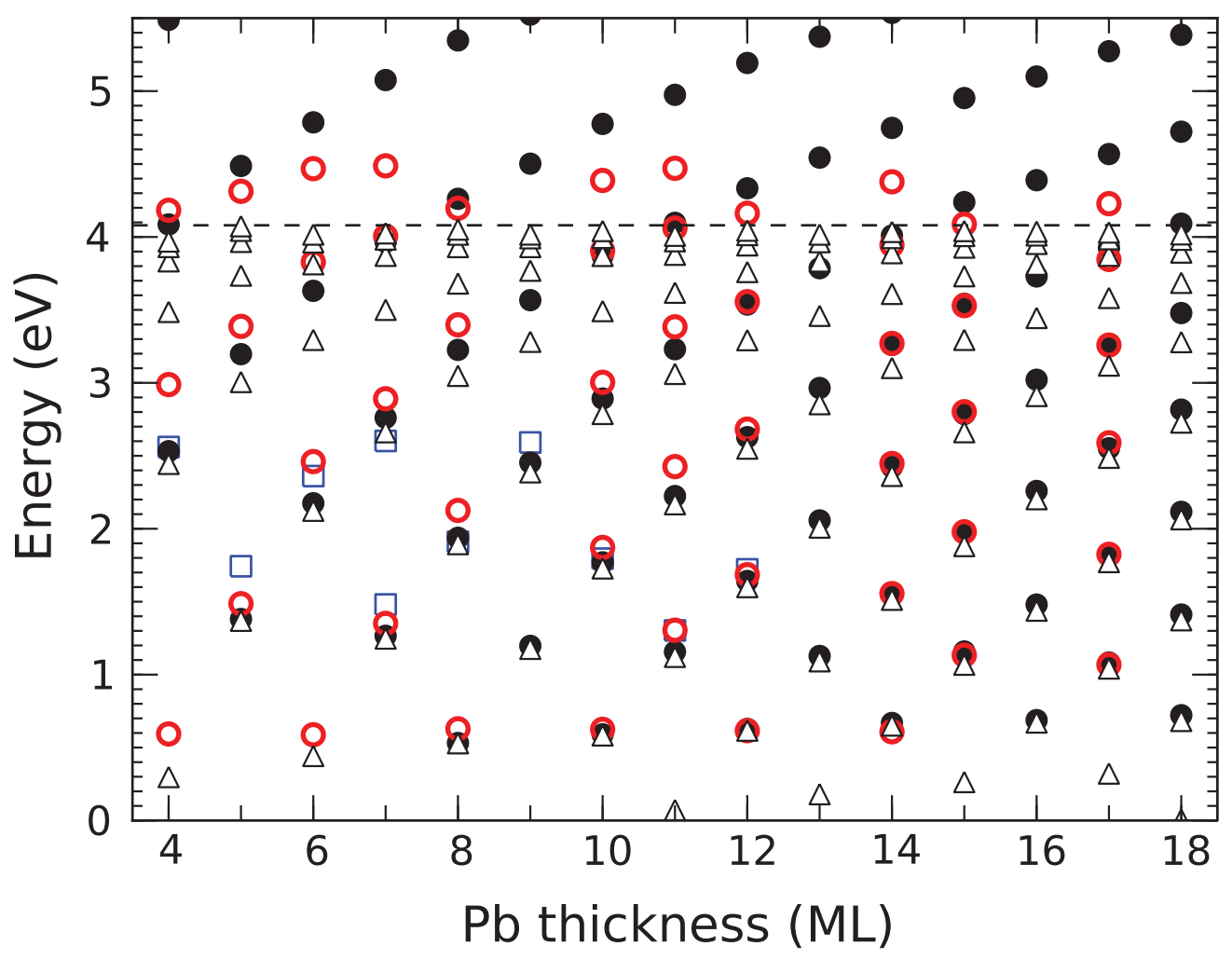

FIG. 9. (Color online) QWS energies at $\bar{\Gamma}$. Results are shown as a function of overlayer thickness. Full dots: calculated energies of the QWS peaks in the $d Z / d V$ spectra. Open dots: experimental QWS peak positions obtained from the $d Z / d V$ spectra of Ref. 21. Open squares: the two-photon photoemission data. ${ }^{23}$ Theoretical results for the bare $\mathrm{Pb} / \mathrm{Cu}(111)$ system (without tip) are shown with open triangles. The horizontal dashed line denotes the vacuum level (work function $\Phi=4.08 \mathrm{eV}$ ).

experimental STS peaks resembles that of the ISs that level off at vacuum level. This interpretation was used by the authors of Ref. 21. Similarly, authors of Ref. 22 analyzed their data within the phase accumulation model with the scattering phase at the $\mathrm{Pb}$ /vacuum interface given by Eq. (16). Thus, it has been assumed that an electron moving in the vacuum region of the STM junction is subject to the image potential only. However, according to our results this explanation does not hold. First, it places the vacuum level at $4.6 \mathrm{eV}$ which is inconsistent with experimental data and ab initio calculations that report a $\mathrm{Pb}$ work function of $4.25 \mathrm{eV}$ at most. ${ }^{35,42,46,77-79}$ The work function oscillations with film thickness can not explain this difference. These are of the order of $\pm 0.1 \mathrm{eV}$ for thin films and become much smaller with increasing coverage. ${ }^{35,79}$ Second, and most important, it follows from the present results that the electric field in the STM junction destroys the ISs-like series causing the Stark energy shift of the QWSs. This effect is well documented in the literature on the example of the ISs transformation into FERs. ${ }^{59-66}$ The vacuum potential barrier becomes essentially triangular as known for FERs, with the corresponding scattering phase shift given by Eq. (17).

The question naturally arises: why the theoretical model does a good job in describing the experimental data up to $\approx 4 \mathrm{eV}$ with respect to the Fermi level and fails for the higher energies? This can not be the deficiency of the modeling of the STM junction. Indeed, reproducing the peaks in the STS spectra up to $\approx 4 \mathrm{eV}$ implies reproducing the Stark shift and so the potential in the junction. At this point it is useful to recall Eq. (15) that defines the energies of the quantized states in the framework of the phase accumulation model. It is reproduced here for convenience: $2 \pi n=2 k\left(E_{n}\right) D+\varphi_{\mathrm{Cu}}\left(E_{n}\right)+\varphi_{\mathrm{vac}}\left(E_{n}\right)$. Provided the nearly free-electron $\mathrm{Cu}(111)$ band structure in this energy range, the $4.6 \mathrm{eV}$ "anomaly" is not linked with $\varphi_{\mathrm{Cu}}\left(E_{n}\right)$. It can be then explained by the energy dependence of the vacuum phase shift $\varphi_{\text {vac }} \cdot{ }^{21}$ Alternatively, it can be associated with $\mathrm{Pb}(111)$ band structure $k\left(E_{n}\right)$ along the direction perpendicular to the film. ${ }^{67}$ Since the former explanation was ruled out, it is reasonable to expect that the disagreement between calculated and experimental data is directly related to the present free-electron description of the overlayer. In the limit of the bulk $\mathrm{Pb}$, the DFT-derived potential for the $\mathrm{Pb}$ film results in the

$$
E=k^{2} / 2-E_{0}
$$

energy dispersion of the states with $k$ along $\Gamma$-L direction. $E_{0} \approx 13.55 \mathrm{eV}$ is the $\mathrm{Pb}$ band bottom. When compared to the ab initio band structure of $\mathrm{Pb}(111)^{41,44,46}$ (see Fig. 1), this free-electron model breaks exactly within the $4-5 \mathrm{eV}$ energy range where different $\mathrm{Pb}$ bands experience avoided crossing. The ab initio result shows $d E / d k \rightarrow 0$ for $k \rightarrow \Gamma$, which would explain the observed experimental trend and the difference with the calculated data.

The direct evidence of the $\mathrm{Pb}(111)$ band structure effect is obtained with the following data analysis based on the phase 

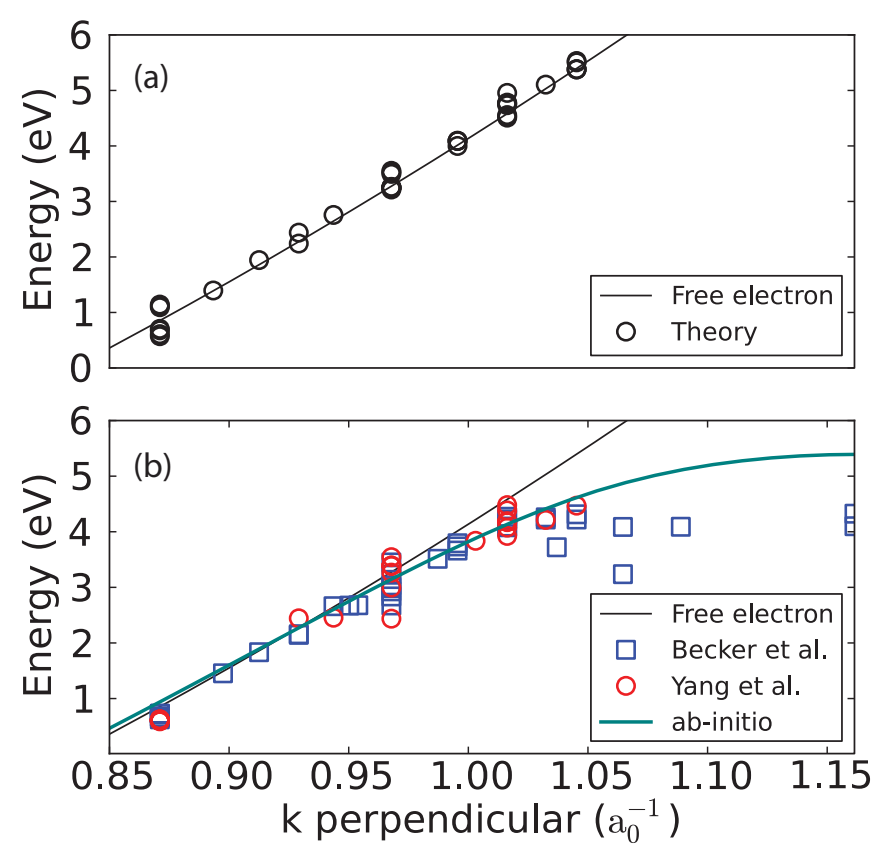

FIG. 10. (Color online) Electronic bulk $\mathrm{Pb}$ band structure along symmetry direction $\Gamma-\mathrm{L}$ in the extended representation. The thin solid curve in both panels corresponds to the free-electron parabolic dispersion given by Eq. (19). (a) Open dots: dispersion derived with Eq. (20) from calculated energies of the STS peaks (see Fig. 9). (b) Open dots: dispersion extracted from experimental data reported for $\mathrm{Pb} / \mathrm{Cu}(111)$ in Ref. 21. Rectangles: dispersion extracted from experimental data reported for $\mathrm{Pb} / \mathrm{Ag}(111)$ in Ref. 22. The relativistic $a b$ initio DFT-LDA dispersion of the $p_{z}$ band of bulk $\mathrm{Pb}^{44}$ is represented with thick blue curve.

accumulation model. ${ }^{22}$ Assume that for the two overlayers with thickness $D_{1}$ and $D_{2}$ one can find the respective spectroscopic peaks $E_{n_{1}}$ and $E_{n_{2}}$ such that $E_{n_{1}}=E_{n_{2}}=E$. Then, subtracting Eq. (15) for the $D_{1}, n_{1}$-case from that for the $D_{2}, n_{2}$-case allows, within the first approximation, to remove the contribution of the scattering phase shifts due to the interfaces. The parent bulk band dispersion along $\Gamma$-L can be then obtained as

$$
k(E)=\pi\left(n_{2}-n_{1}\right) /\left(D_{2}-D_{1}\right) .
$$

However, when performing constant current spectroscopy for the overlayers with different thickness $D_{2}>D_{1}$, the tip-surface distance $Z$ is not the same when probing the states at the same energy. This is because the number of the electronic states to tunnel to is higher for the thicker overlayer, which has to be compensated with increasing $Z$. For the same bias, the electric field in a junction is then smaller for $D_{2}$, and the cancelation of the vacuum phase shifts is an approximation. To access the validity of this approximation, we have first transformed the calculated data according to Eq. (20). The STS peaks were assumed to have the same energy if they fall within a $40-\mathrm{meV}$ energy window: $\left|E_{n_{1}}-E_{n_{2}}\right|<40 \mathrm{meV}$. The extracted band dispersion is shown in Fig. 10(a). It appears very close to the free-electron parabola given by Eq. (19), so that the $k(E)$ extraction procedure holds.

Having checked the validity of the method outlined above, we applied it to the experimental data of Ref. 21. The ensuing
$\mathrm{Pb}(111)$ band dispersion along $\Gamma-\mathrm{L}$ is presented in Fig. 10(b) together with the $a b$ initio DFT-LDA relativistic calculations of the $\mathrm{Pb}$ band structure. We also plot the $k(E)$ derived by the same method for $\mathrm{Pb} / \mathrm{Ag}(111)$ as published in Ref. 22. In sheer contrast with the free-electron parabola, the $a b$ initio band dispersion saturates at $\Gamma$ point for $E-E_{f}=5.4 \mathrm{eV}$ and for $k=1.161 a_{0}^{-1}$ (reciprocal lattice vector). The dispersion relation extracted from STS experiments agrees with $a b$ initio results and photoemission data. ${ }^{46}$ This fully validates our interpretation of the experimentally observed trends in the QWSs series at high bias as a direct consequence of the realistic band structure of $\mathrm{Pb}(111)$. Obviously, it can not be reproduced with the present approach based on the free-electron modeling of $\mathrm{Pb}$ overlayer, which explains disagreement between calculated and measured STS peak positions at bias above $4 \mathrm{eV}$.

Recently, a larger set of the STS spectra for $\mathrm{Pb} / \mathrm{Cu}(111)$ has been shown with bias range extending up to $9.0 \mathrm{eV} .{ }^{89}$ The FERs with energies independent of the $\mathrm{Pb}$ film thickness were reported for the bias above $5 \mathrm{eV}$. According to the model results of present work, the free-electron-like description of $\mathrm{Pb}$ would not explain the experimental data since we obtain thicknessdependent peak energies at high bias. While the detailed discussion is beyond the scope of this paper, the nontrivial $\mathrm{Pb}$ band structure above $4.5 \mathrm{eV}$, as shown in Fig. 1, should be at the origin of the observed effects. Several possible explanations, all based on the $\mathrm{Pb}$ band structure effect, can be advanced: (i) increased surface reflectivity in the corresponding energy range, (ii) flat dispersion of the underlying $\mathrm{Pb}$ bands, (iii) high electron transmission through the $\mathrm{Pb} / \mathrm{Cu}(111)$ interface above the projected band gap of $\mathrm{Cu}(111)$, so that the electron does not return to the $\mathrm{Pb}$ /vacuum interface and all confinement is at the vacuum side.

\section{SUMMARY AND CONCLUSIONS}

We have performed a theoretical study of the constant current scanning-tunneling spectroscopy of thin $\mathrm{Pb}$ films on a $\mathrm{Cu}(111)$ substrate. The $d Z / d V$ spectra have been calculated for the 4-18 ML film thickness range.

The tip-sample system is represented within the flat-tip approximation, so that well-developed approaches for modeling the STM junction ${ }^{62}$ could be applied. The $1 \mathrm{D} \mathrm{Pb} / \mathrm{Cu}(111)$ potential has been derived from self-consistent 1D DFTLDA calculations, allowing an adequate description of both electronic structure and dynamical properties of $\mathrm{Pb}$ overlayers on $\mathrm{Cu}(111) .{ }^{37,48,50} \mathrm{We}$ explicitly account for the projected band gap (L gap) of the $\mathrm{Cu}(111)$ substrate at $\bar{\Gamma}$. The $\mathrm{Pb}$ overlayer is described within the free-electron (jellium) model. Provided the tip- $\mathrm{Pb} / \mathrm{Cu}(111)$ potential, the electron transmission through the junction is obtained at $\bar{\Gamma}$ using the WPP method. The manybody inelastic effects (electron-electron and electron-phonon energy relaxations) are accounted for with a bias-dependent absorbing potential in the sample.

Within the studied range of overlayer thicknesses, the energies of the calculated peaks in the STS spectra reproduce the experimental data for bias below $4 \mathrm{eV}$. Particularly remarkable agreement with respect to the energies, widths, and relative intensities of the STS peaks is obtained for thicknesses above $11 \mathrm{ML}$, i.e., in the range where the quantum size effects 
on the $\mathrm{Pb}$ film work function and the effect of the wetting layer can be considered as negligible.

Simultaneous calculations of the (i) $d Z / d V$ spectra, (ii) quasistationary QWSs in the tip-sample system at fixed tipsample distance and bias, and (iii) QWSs of bare $\mathrm{Pb} / \mathrm{Cu}(111)$ without the tip have been performed. This allows detailed discussion of the physics underlying experimental observations. It appears that only low-energy QWSs are unperturbed by the tip. At high bias, the tip-induced Stark shift determines the energies of the QWSs and so the peaks in the STS spectra. Here, the effect of the electric field in the junction is similar to that often evoked in discussion of the transformation of ISs into FERs. ${ }^{63,64}$ Indeed, the electronic density of the high-energy QWSs in bare $\mathrm{Pb} / \mathrm{Cu}(111)$ system is shifted from the inside $\mathrm{Pb}$ film into the vacuum, indicating partial IS character. ${ }^{83}$

Good agreement between the calculated and measured data in the energy range corresponding to the free-electron-like dispersion of the $\mathrm{Pb}$ band along $\Gamma$-L direction validates the present theoretical approach. The departure of the experimentally measured energies of the STS peaks from the theoretical results above $4 \mathrm{eV}$ bias is then sound. It reflects the departure of the bulk $\mathrm{Pb}$ band structure along $\Gamma$-L from free-electron parabola. Comparison between the $a b$ initio band structure of $\mathrm{Pb}$, the two-photon photoemission data, and $k(E)$ dispersion curves extracted from experimental data of Refs. 21 and 22 fully confirms the above interpretation. Thus, the STS performed at different coverages can be used as a complementary tool to inverse photoemission to probe the dispersion of the bulk bands of the overlayer material at energies well above the Fermi level.

We believe that the present theoretical study contributes to the understanding of STM experiments performed on QWSs in $\mathrm{Pb}$ overlayers supported on metallic surfaces like $\mathrm{Cu}(111)$ and $\operatorname{Ag}(111)$. It also sheds light on some fundamental issues present in STS studies of metallic films supported on different substrates.

\section{APPENDIX: CALCULATION OF $d Z / d V$ CHARACTERISTIC}

Here, we discuss the numerical procedure used to calculate the constant current $d Z / d V$ characteristic. At large enough tip-sample distances, we assume for the tip position a wellbehaved function of electric current $I$ and bias voltage $V$, i.e.,
$Z \equiv Z(I, V)$, thus

$$
d Z=\left(\frac{\partial Z}{\partial V}\right)_{I} d V+\left(\frac{\partial Z}{\partial I}\right)_{V} d I .
$$

Assuming small $\delta V$ and $\delta I$ variations and recasting (A1) together with initial conditions, if necessary, determined by a simple root-finding bisection method applied to the equation $I(V, Z)=I_{0}$,

$$
\begin{gathered}
Z_{0}^{0} \equiv Z\left(V_{0}, I_{0}+\delta I_{0}\right), \\
Z_{0}^{1} \equiv Z\left(V_{0}, I_{0}\right), \\
Z_{1}^{0} \equiv Z\left(V_{0}+\delta V_{0}, I_{0}\right),
\end{gathered}
$$

we calculate iteratively the corresponding $Z\left(V_{j}, I_{0}\right) \approx Z_{j}^{i_{c}}=$ $Z_{j-1}^{i_{c}}+\sum_{i=0}^{i_{c}} \delta Z_{j}^{i}$ for fixed $I_{0}$ and each $V_{j}(j=2,3, \ldots)$. The index value $i_{c}$ corresponds to the $i$ th iteration for which convergence is reached, defined by the convergence condition $\left|I^{i_{c}}-I_{0}\right| / I_{0}<\Delta_{c}$. We obtain $Z_{j}^{0}=Z_{j-1}^{i_{c}}+\delta Z_{j}^{0}$ with

$$
\delta Z_{j}^{0}=\left(\frac{Z_{j-1}^{i_{c}}-Z_{j-2}^{i_{c}}}{V_{j-1}-V_{j-2}}\right) \delta V_{j}
$$

and $Z_{j}^{i}=Z_{j}^{i-1}+\delta Z_{j}^{i}$ (for $i_{c}>0$ ) with

$$
\delta Z_{j}^{i}=\left(\frac{Z_{k}^{i_{c}}-Z_{k}^{i_{c}-1}}{I_{k}^{i_{c}}-I_{k}^{i_{c}-1}}\right)\left(I_{0}-I_{j}^{i-1}\right),
$$

where the $k$ index refers to the highest $k<j$ for which $i_{c}>0$. If $i_{c}>1$, the following might be used for $i$ running from 2 to $i_{c}$ :

$$
\delta Z_{j}^{i}=\left(\frac{Z_{j}^{i-1}-Z_{j}^{i-2}}{I_{j}^{i-1}-I_{j}^{i-2}}\right)\left(I_{0}-I_{j}^{i-1}\right) .
$$

We have performed most of the calculations with $\Delta_{c}=$ 0.001 and constant $\delta V_{j}=50 \mathrm{meV}$. The latter value should be taken with care because resonant peak energies converge faster than linewidths. With these values, for most $j, i_{c}=1$ (two calculation per $V_{j}$ ). The above procedure is essentially the application of the Newton-Raphson method [Eq. (A5)] to the equation $I\left(V_{j}, Z\right)-I_{0}=0$ with an efficient prescription [Eqs. (A3) and (A4)] for the initial guess of the root $Z_{j}$.

Finally, the constant current $d Z / d V$ spectra are obtained by numerical differentiation of the calculated $Z-V$ characteristic.
${ }^{1}$ F. J. García de Abajo, J. Cordón, M. Corso, F. Schiller, and J. E. Ortega, Nanoscale 2, 717 (2010).

${ }^{2}$ M. F. Crommie, C. P. Lutz, and D. M. Eigler, Science 262, 218 (1993).

${ }^{3}$ K.-F. Braun and K.-H. Rieder, Phys. Rev. Lett. 88, 096801 (2002).

${ }^{4}$ V. S. Stepanyuk, L. Niebergall, W. Hergert, and P. Bruno, Phys. Rev. Lett. 94, 187201 (2005).

${ }^{5}$ J. Li, Wolf-Dieter Schneider, R. Berndt, and S. Crampin, Phys. Rev. Lett. 80, 3332 (1998).

${ }^{6}$ H. Jensen, J. Kröger, R. Berndt, and S. Crampin, Phys. Rev. B 71, 155417 (2005).
${ }^{7}$ L. Niebergall, G. Rodary, H. F. Ding, D. Sander, V. S. Stepanyuk, P. Bruno, and J. Kirschner, Phys. Rev. B 74, 195436 (2006).

${ }^{8}$ K. Schouteden and C. Van Haesendonck, Phys. Rev. Lett. 103, 266805 (2009).

${ }^{9}$ C. Tournier-Colletta, B. Kierren, Y. Fagot-Revurat, and D. Malterre, Phys. Rev. Lett. 104, 016802 (2010)

${ }^{10}$ F. Klappenberger, D. Kühne, W. Krenner, I. Silanes, A. Arnau, F. J. García de Abajo, S. Klyatskaya, M. Ruben, and J. V. Barth, Phys. Rev. Lett. 106, 026802 (2011).

${ }^{11}$ N. Nilius, T. M. Wallis, and W. Ho, Science 297, 1853 (2002).

${ }^{12}$ S. Fölsch, P. Hyldgaard, R. Koch, and K. H. Ploog, Phys. Rev. Lett. 92, 056803 (2004) 
${ }^{13}$ J. Lagoute, C. Nacci, and S. Fölsch, Phys. Rev. Lett. 98, 146804 (2007)

${ }^{14}$ Y. Pennec, W. Auwärter, A. Schiffrin, A. Weber-Bargioni, A. Riemann, and J. V. Barth, Nat. Nanotechnology 2, 99 (2007).

${ }^{15}$ C. Chiang, Surf. Sci. Rep. 39, 181 (2000).

${ }^{16}$ M. Milun, P. Pervan, and D. P. Woodruff, Rep. Prog. Phys. 65, 99 (2002).

${ }^{17}$ Q. Qiu and N. V. Smith, J. Phys. Condens. Matter 14, R169 (2002).

${ }^{18}$ C. Corriol, V. M. Silkin, D. Sánchez-Portal, A. Arnau, E. V. Chulkov, P. M. Echenique, T. von Hofe, J. Kliewer, J. Kröger, and R. Berndt, Phys. Rev. Lett. 95, 176802 (2005).

${ }^{19}$ J. H. Dil, J. Phys. Condens. Matter 21, 403001 (2009).

${ }^{20}$ T. Miller, A. Samsavar, G. E. Franklin, and T.-C. Chiang, Phys. Rev. Lett. 61, 1404 (1988).

${ }^{21}$ M. C. Yang, C. L. Lin, W. B. Su, S. P. Lin, S. M. Lu, H. Y. Lin, C. S. Chang, W. K. Hsu, and Tien T. Tsong, Phys. Rev. Lett. 102, 196102 (2009).

${ }^{22}$ M. Becker and R. Berndt, Phys. Rev. B 81, 205438 (2010).

${ }^{23} \mathrm{~S}$. Mathias, A. Ruffing, F. Deicke, M. Wiesenmayer, M. Aeschlimann, and M. Bauer, Phys. Rev. B 81, 155429 (2010).

${ }^{24}$ P. M. Echenique and J. B. Pendry, J. Phys. C 11, 2065 (1978).

${ }^{25}$ E. G. McRae and M. L. Kane, Surf. Sci. 108, 435 (1981).

${ }^{26}$ N. V. Smith, Phys. Rev. B 32, 3549 (1985).

${ }^{27}$ S. Crampin, S. De Rossi, and F. Ciccacci, Phys. Rev. B 53, 13817 (1996).

${ }^{28}$ J. J. Paggel, T. Miller, and T.-C. Chiang, Science 283, 1709 (1999).

${ }^{29}$ F. Schiller, A. Leonardo, E. V. Chulkov, P. M. Echenique, and J. E. Ortega, Phys. Rev. B 79, 033410 (2009).

${ }^{30}$ C. M. Wei, and M. Y. Chou, Phys. Rev. B 66, 233408 (2002).

${ }^{31}$ X. Ma, P. Jiang, Y. Qi, J. Jia, Y. Yang, W. Duan, W.-X. Li, X. Bao, S. B. Zhang, and Qi-Kun Xue, PNAS 104, 9204 (2007).

${ }^{32}$ Y.-F. Zhang, J.-F. Jia, T.-Z. Han, Z. Tang, Q.-T. Shen, Y. Guo, Z. Q. Qiu, and Q.-K. Xue, Phys. Rev. Lett. 95, 096802 (2005).

${ }^{33}$ S. Mathias, M. Wiesenmayer, M. Aeschlimann, and M. Bauer, Phys. Rev. Lett. 97, 236809 (2006).

${ }^{34}$ C. Brun, I-Po Hong, F. Pattley, I. Y. Sklyadneva, R. Heid, P. M. Echenique, K. P. Bohnen, E. V. Chulkov, and W.-D. Schneider, Phys. Rev. Lett. 102, 207002 (2009).

${ }^{35}$ Y. Jia, B. Wu, H. H. Weitering, and Z. Zhang, Phys. Rev. B 74, 035433 (2006).

${ }^{36}$ A. L. Vázquez de Parga, J. J. Hinarejos, F. Calleja, J. Camarero, R. Otero, and R. Miranda, Surf. Sci. 603, 1389 (2009).

${ }^{37}$ E. Ogando, N. Zabala, E. V. Chulkov, and M. J. Puska, Phys. Rev. B 69, 153410 (2004).

${ }^{38}$ P. S. Kirchmann and U. Bovensiepen, Phys. Rev. B 78, 035437 (2008).

${ }^{39}$ P. S. Kirchmann, L. Rettig, X. Zubizarreta, V. M. Silkin, E. V. Chulkov, and U. Bovensiepen, Nat. Phys. 6, 782 (2010).

${ }^{40}$ S. M. Lu, W. B. Su, C. L. Lin, W. Y. Chan, H. L. Hsiao, C. S. Chang, and T. T. Tsong, J. Appl. Phys. 108, 083707 (2010).

${ }^{41}$ I-Po Hong, C. Brun, F. Patthey, I. Yu. Sklyadneva, X. Zubizarreta, R. Heid, V. M. Silkin, P. M. Echenique, K. P. Bohnen, E. V. Chulkov, and W-D. Schneider, Phys. Rev. B 80, 081409 (2009).

${ }^{42}$ C. M. Wei, and M. Y. Chou, Phys. Rev. B 75, 195417 (2007).

${ }^{43}$ A. Ayuela, E. Ogando, and N. Zabala, Phys. Rev. B 75, 153403 (2007).

${ }^{44}$ X. Zubizarreta, V. M. Silkin, and E. V. Chulkov, Accepted (Jul 25 2011) for publication in Phys. Rev B.
${ }^{45}$ E. V. Chulkov, V. M. Silkin, and P. M. Echenique, Surf. Sci. 437, 330 (1999).

${ }^{46}$ K. Horn, B. Reihl, A. Zartner, D. E. Eastman, K. Hermann, and J. Noffke, Phys. Rev. B 30, 1711 (1984).

${ }^{47}$ H. Eckardt, L. Fritsche, and J. Noffke, J. Phys. F 14, 97 (1984).

${ }^{48}$ E. Ogando, N. Zabala, E. V. Chulkov, and M. J. Puska, Phys. Rev. B 71, 205401 (2005).

${ }^{49}$ E. Ogando, N. Zabala, E. V. Chulkov, and M. J. Puska, J. Phys. Condens. Matter 20, 315002 (2008)

${ }^{50}$ A. Zugarramurdi, N. Zabala, V. M. Silkin, A. G. Borisov, and E. V. Chulkov, Phys. Rev. B 80, 115425 (2009).

${ }^{51}$ R. Otero, A-L. Vázquez de Parga, and R. Miranda, Phys. Rev. B 66, 115401 (2002).

${ }^{52}$ A. Weismann, M. Wenderoth, S. Lounis, P. Zahn, N. Quaas, R. G. Ulbrich, P. H. Dederichs, and S. Blügel, Science 323, 1190 (2009).

${ }^{53}$ J. Li, W. D. Schneider, R. Berndt, O. R. Bryant, and S. Crampin, Phys. Rev. Lett. 81, 4464 (1998).

${ }^{54}$ J. Kliewer, R. Berndt, E. V. Chulkov, V. M. Silkin, P. M. Echenique, and S. Crampin, Science 288, 1399 (2000).

${ }^{55}$ E. V. Chulkov, J. Kliewer, R. Berndt, V. M. Silkin, B. Hellsing, S. Crampin, and P. M. Echenique, Phys. Rev. B 68, 195422 (2003).

${ }^{56}$ M. A. Schneider, L. Vitali, N. Knorr, and K. Kern, Phys. Rev. B 65 , 121406(R) (2002).

${ }^{57}$ U. Höfer, I. L. Shumay, C. Reuß, U. Thomann, W. Wallauer, and T. Fauster, Science 277, 1480 (1997).

${ }^{58}$ T. Fauster, C. Reuß, I. L. Shumay, and M. Weinelt, Chem. Phys. 251, 111 (2000).

${ }^{59}$ G. Binnig, K. H. Frank, H. Fuchs, N. Garcia, B. Reihl, H. Rohrer, F. Salvan, and A. R. Williams, Phys. Rev. Lett. 55, 991 (1985).

${ }^{60}$ R. S. Becker, J. A. Golovchenko, and B. S. Swartzentruber, Phys. Rev. Lett. 55, 987 (1985).

${ }^{61}$ J. H. Coombs, and J. K. Gimzewski, J. Microsc. 152, 841 (1988).

${ }^{62}$ J. M. Pitarke, F. Flores, and P. M. Echenique, Surf. Sci. 234, 1 (1990).

${ }^{63}$ P. Wahl, M. A. Schneider, L. Diekhöner, R. Vogelgesang, and K. Kern, Phys. Rev. Lett. 91, 106802 (2003).

${ }^{64}$ A. Hanuschkin, D. Wortmann, and S. Blügel, Phys. Rev. B 76, 165417 (2007).

${ }^{65}$ D. B. Dougherty, P. Maksymovych, J. Lee, M. Feng, H. Petek, and J. T. Yates Jr., Phys. Rev. B 76, 125428 (2007).

${ }^{66}$ J. I. Pascual, C. Corriol, G. Ceballos, I. Aldazabal, H.-P. Rust, K. Horn, J. M. Pitarke, P. M. Echenique, and A. Arnau, Phys. Rev. B 75, 165326 (2007).

${ }^{67}$ A. Zugarramurdi, N. Zabala, A. G. Borisov, and E. V. Chulkov, Phys. Rev. Lett. 106, 249601 (2011).

${ }^{68}$ Numerical Grid Methods and Their Application to Schrödinger's Equation, NATO ASI Series, Series C: Mathematical and Physical Sciences, edited by C. Cerjan (Kluwer Academic Publishers, London, 1993), Vol. 412.

${ }^{69}$ E. V. Chulkov, A. G. Borisov, J. P. Gauyacq, D. Sánchez-Portal, V. M. Silkin, V. P. Zhukov, and P. M. Echenique, Chem. Rev. 106, 4160 (2006).

${ }^{70}$ D. Neuhauser, and M. Baer, J. Chem. Phys. 91, 4651 (1989).

${ }^{71}$ M. A. Van Hove, W. H. Weinberg and C. M. Chan, Low-Energy Electron Diffraction (Springer-Verlag, Berlin, 1986).

${ }^{72}$ C. Leforestier, R. H. Bisseling, C. Cerjan, M. D. Feit, R. Friesner, A. Guldberg, A. Hammerich, G. Jolicard, W. Karrlein, H. D. Meyer, N. Lipkin, O. Roncero, and R. Kosloff, J. Comput. Phys. 94, 59 (1991). 
${ }^{73}$ M. D. Feit, J. A. Fleck Jr., and A. Steiger, J. Comput. Phys. 47, 412 (1982).

${ }^{74}$ D. Kosloff and R. Kosloff, J. Comput. Phys. 52, 35 (1983).

${ }^{75}$ J. Sjakste, A. G. Borisov, J. P. Gauyacq, and A. K. Kazansky, J. Phys. B 37, 1593 (2004).

${ }^{76}$ C. B. Duke, Tunneling in Solids, Solid State Physics: Advances in Research and Applications, Suppl. No. 10 (Academic Press, New York, 1969).

${ }^{77}$ D. Yu and M. Scheffler, Phys. Rev. B 70, 155417 (2004).

${ }^{78}$ K. Jacobi, Phys. Rev. B 38, 5869 (1988).

${ }^{79}$ P. S. Kirchmann, M. Wolf, J. H. Dil, K. Horn, and U. Bovensiepen, Phys. Rev. B 76, 075406 (2007).

${ }^{80}$ A. Kiejna, Phys. Rev. B 47, 7361 (1993).

${ }^{81}$ R. O. Jones, P. J. Jennings, and O. Jepsen, Phys. Rev. B 29, 6474 (1984).
${ }^{82}$ J. Quinn and R. Ferell, Phys. Rev. 112, 812 (1958).

${ }^{83}$ R. Fischer and Th. Fauster, Phys. Rev. B 51, 7112 (1995).

${ }^{84}$ S. Ogawa, S. Heike, H. Takahashi, and T. Hashizume, J. Appl. Phys. 101, 09G504 (2007).

${ }^{85}$ S. Ogawa, S. Heike, H. Takahashi, and T. Hashizume, Phys. Rev. B 75, 115319 (2007)

${ }^{86}$ S. Crampin, Phys. Rev. Lett. 95, 046801 (2005).

${ }^{87}$ A. J. Caamaño, Y. Pogorelov, O. Custance, J. Méndez, A. M. Baró, J. Y. Veuillen, J. M. Gómez-Rodríguez, and J. J. Sáenz, Surf. Sci. 426, L420 (1999).

${ }^{88}$ Handbook of Mathematical Functions, edited by M. Abramowitz and I. A. Stegun (Dover, Mineola, NY, 1972).

${ }^{89}$ C. L. Lin, M. C. Yang, W. B. Su, S. P. Lin, S. M. Lu, H. Y. Lin, C. S. Chang, T. Y. Fu, and Tien T. Tsong, Chin. J. Phys. 48, 855 (2010). 